\title{
PSEUDO TRANSIENT CONTINUATION AND TIME MARCHING METHODS FOR MONGE-AMPÈRE TYPE EQUATIONS
}

\author{
GERARD AWANOU
}

\begin{abstract}
We present two numerical methods for the fully nonlinear elliptic MongeAmpère equation. The first is a pseudo transient continuation method and the second is a pure pseudo time marching method. The methods are proven to converge to a strictly convex solution of a natural discrete variational formulation with $C^{1}$ conforming approximations. The assumption of existence of a strictly convex solution to the discrete problem is proven for smooth solutions of the continuous problem and supported by numerical evidence for non smooth solutions.
\end{abstract}

\section{INTRODUCTION}

We are interested in numerical solutions of the fully nonlinear elliptic Monge-Ampère equation

$$
\operatorname{det} D^{2} u=f \text { in } \Omega, \quad u=g \text { on } \partial \Omega,
$$

on a convex bounded domain $\Omega$ of $\mathbb{R}^{n}, n=2,3$ with boundary $\partial \Omega$. The unknown $u$ is a real valued function and $f, g$ are given functions with $f>0$ in the non degenerate case and $f \geq 0$ in the degenerate case. We will also assume that $f \in C(\Omega)$ and $g$ in $C(\partial \Omega)$.

Starting with [8, 15], interest has grown for finite element methods which are able to capture non smooth solutions of second order fully nonlinear equations. For smooth solutions, the problem was studied in the context of semiconforming $C^{1}$ finite elements by Böhmer [9, 10] on both smooth and polygonal domains. Böhmer addressed general fully nonlinear elliptic equations for the first time. Brenner et al [12] used Lagrange elements and interior penalty terms on smooth domains. Non smooth solutions can be handled with finite elements in the context of the vanishing moment methodology [22], a singular perturbation of (1.1). Proven convergence methods for non smooth solutions include the work of Oliker and Prussner [31], Feng and Neilan [20] for radial viscosity solutions in the finite element context and the work of Oberman [30] who addressed in general functions of the eigenvalues of the Hessian in the context of monotone finite difference methods. For recent developments we refer to [19].

In this paper we give numerical evidence that $C^{1}$ conforming approximations of a natural variational formulation of (1.1) converge for non smooth solutions of the two dimensional problem. This is achieved by discretizing new iterative methods we introduce. We establish the convergence of the iterative methods under the assumption that the discrete problem has a strictly convex solution. We prove that such a solution exists when (1.1) has a smooth strictly convex solution. We do not assume that (1.1) has a smooth solution for our iterative methods to converge. The existence of a 
convex solution to the discrete problem in the general case and the convergence of the discretization will be addressed in a subsequent paper. Even with such an existence result, it is still a non trivial task to solve the discrete nonlinear systems in situations where (1.1) has a non smooth solution. This paper addresses this issue.

The main technical innovation of this paper is the proof that in the context of $C^{1}$ conforming approximations, discrete functions near a strictly convex solution are strictly convex. This explains why convexity did not need to be imposed explicitly in some previous studies. Newton's method remains the most appropriate iterative method for solving the discrete nonlinear equations when (1.1) has a smooth convex solution. We give a new proof of convergence of Newton's method in the context of $C^{1}$ conforming approximations.

The results of this paper extend easily to finite dimensional spaces of piecewise smooth $C^{1}$ functions provided that the approximation property $(2.2)$ and inverse estimates (2.4) below hold.

Our results can be described in the general context of discretizations by $C^{1}$ elements of iterative methods for a general nonlinear elliptic equation $F(u)=0$. In the case of the Monge-Ampère equation, $F(u)=\operatorname{det} D^{2} u-f$. We first describe the iterative methods at the continuous level. However, we will not address convergence at the continuous level. As an initial guess we take the solution of the Poisson equation $\Delta u=n f^{1 / n}$ in $\Omega, u=g$ on $\partial \Omega$.

1.1. Pseudo transient continuation method. We assume that $F$ differentiable and consider the sequence of problems

$$
\left(\nu L+F^{\prime}\left(u_{k}\right)\right)\left(u_{k+1}-u_{k}\right)=-F\left(u_{k}\right),
$$

where $L$ is a linear operator which can be taken as $L=-I$ where $I$ is the identity operator or $L=\Delta$ where $\Delta$ is the Laplace operator and $\nu>0$ is a parameter. Pseudo transient continuation methods [26] form a general class of methods for solving nonlinear singular equations. In the case of the Monge-Ampère equation, the method consists in solving the sequence of approximate problems

$$
\nu L \theta_{k}+\left(\operatorname{cof} D^{2} u_{k}\right): D^{2} \theta_{k}=\left(f-f_{k}\right), f_{k}=\operatorname{det} D^{2} u_{k}, \theta_{k}=u_{k+1}-u_{k} .
$$

Here $\operatorname{cof} A$ denotes the matrix of cofactors of the matrix $A$.

1.2. Pseudo time marching method. Given $\nu>0$, we consider the sequence of iterates

$$
-\nu \Delta u_{k+1}=-\nu \Delta u_{k}+F\left(u_{k}\right), u_{k+1}=g \text { on } \partial \Omega .
$$

This can be interpreted as an Euler discretization of the pseudo time dependent equation $\frac{\partial \Delta u}{\partial t}+F(u)=0$, or as a Laplacian preconditioner of a simple pseudo time marching algorithm, [23] $u_{k+1}=u_{k}-\frac{1}{\nu} \Delta^{-1} F\left(u_{k}\right)$. See also a remark in [29].

To the author's best knowledge, this is the first time the pseudo transient continuation method and the time marching method are used to indicate numerically convergence to viscosity solutions of finite element type methods for the Monge-Ampère equation. 
1.3. Advantages and comparison of the two methods. The methods we propose can be used in the context of different types of discretizations allowing us in particular to treat more easily non-rectangular domains. The methods can be accelerated with fast Poisson solvers and multigrid methods. This latter property is even more striking for the time marching method as its implementation requires only having access to a multigrid Poisson solver.

Although the theory of the Monge-Ampère equation has concentrated on convex solutions, one can equally focus on concave solutions. We found out that (1.3) is better able to capture concave solutions. It is easy to implement, requiring only a Poisson solver. For example one can capture weak solutions of the Monge-Ampere equation by simply discretizing $(1.3)$ with the standard Lagrange finite elements. The time marching method can also be applied to fully nonlinear equations such as the Pucci equation where $F$ is not differentiable.

In summary the pseudo transient continuation methods are better for smooth solutions and singular solutions on a coarse mesh. Otherwise the method of choice is the time marching method.

1.4. Organization of the paper. We organize the paper as follows: in the second section we introduce some notation and prove the key result that discrete functions near a strictly convex solution are strictly convex. We introduce the natural variational formulation of (1.1) and state an existence and uniqueness result for the discrete problem. As a corollary the discrete variational problem has a convex solution when (1.1) has a smooth convex solution. We study the pseudo transient continuation methods in section 3. A special case is Newton's method for which we prove a quadratic convergence rate. The time marching methods are studied in section 4. The last section is devoted to numerical results. We give a brief description of the spline element method which is used for the computations and offer heuristics about why our methods appear to preserve convexity.

\section{Notation AND PRELIMINARIES}

We use the standard notation for the Sobolev spaces $W^{k, p}(\Omega)$ with norms $\|.\|_{k, p}$ and semi-norm $|.|_{k, p}$. In particular, $H^{k}(\Omega)=W^{k, 2}(\Omega)$ and in this case, the norm and seminorms will be denoted respectively by $\|.\|_{k}$ and $|.|_{k}$. For a vector field $v=\left(v_{i}\right)_{i=1, \ldots, d}$ with values in $W^{k, p}(\Omega)^{n}, 1 \leq p<\infty$, we set $\|v\|_{k, p}=\left(\sum_{i=1}^{n}\left\|v_{i}\right\|_{k, p}^{2}\right)^{\frac{1}{2}}$ and a similar notation for $|v|_{k, p}$. In the case $p=\infty$, we set $\|v\|_{k, \infty}=\max _{i=1, \ldots, n}\left\|v_{i}\right\|_{k, \infty}$ with a similar notation for $|v|_{k, \infty}$. For matrix valued fields, the above notation is extended canonically.

We make the usual convention of denoting constants by $C$ but will occasionally index some constants.

We make the assumption that the boundary of $\Omega$ is polygonal and that the triangulation $\mathcal{T}$ is shape regular in the sense that there is a constant $C>0$ such that for any triangle $K, h_{K} / \rho_{K} \leq C$, where $h_{K}$ denotes the diameter of $K$ and $\rho_{K}$ the radius of the largest ball contained in $K$. We also require the triangulation to be quasi-uniform in 
the sense that $h / h_{\text {min }}$ is bounded where $h$ and $h_{\text {min }}$ are the maximum and minimum respectively of $\left\{h_{K}, K \in \mathcal{T}_{h}\right\}$.

We define

$$
V^{h}:=S_{d}^{1}(\mathcal{T})=\left\{s \in C^{1}(\Omega),\left.s\right|_{t} \in \mathcal{P}_{d}, \forall t \in \mathcal{T}\right\},
$$

where $\mathcal{P}_{d}$ denotes the space of polynomials of degree less than or equal to $d$.

In two dimensions, it is known that, [28], for $d \geq 5$ and $0 \leq l \leq d$, there exists a linear quasi-interpolation operator $Q_{h}$ mapping $L_{1}(\Omega)$ into the spline space $S_{d}^{1}(\mathcal{T})$ and a constant $C$ such that if $v$ is in the Sobolev space $W^{l+1, p}(\Omega), 1 \leq p \leq \infty$

$$
\left\|v-Q_{h} v\right\|_{k, p} \leq C h^{l+1-k}|v|_{l+1, p},
$$

for $0 \leq k \leq l$. If $\Omega$ is convex, the constant $C$ in $(2.2)$ depends only on $d, l$ and on the smallest angle $\theta_{h}$ in $\mathcal{T}$. In the nonconvex case, $C$ depends only on the Lipschitz constant associated with the boundary of $\Omega$. It is also known c.f. [16] that the full approximation property for spline spaces holds on special triangulations for certain values of $d$.

In three dimensions, 2.2 holds in general for $d \geq 9$, c.f. [28].

Note that, by 2.2,

$$
\left\|Q_{h} v\right\|_{2, p} \leq C\|v\|_{2, p}, \quad v \in W^{2, p}(\Omega)
$$

for all $p \geq 1$.

We assume that the following inverse inequality holds

$$
\|v\|_{s, p} \leq C h^{l-s+\min \left(0, \frac{n}{p}-\frac{n}{q}\right)}\|v\|_{l, q}, \forall v \in V^{h}
$$

for $0 \leq l \leq s, 1 \leq p, q \leq \infty$. For $C^{1}$ finite element spaces the result can be found in [14], Theorem 4.5.11. For the spline spaces they may be viewed as a consequence of the assumption of uniform triangulation and of Markov inequality, 28] p. 2. See also [10], section 4.2.6.

2.1. Variational formulations. We first recall the divergence form of the determinant and the expression of its Fréchet derivative.

For two $n \times n$ matrices $M, N$, we recall the Frobenius product

$$
M: N=\sum_{i, j=1}^{n} M_{i j} N_{i j} .
$$

In particular, for a matrix $A$, we have $(\operatorname{cof} A): A=\sum_{i, j=1}^{n}(\operatorname{cof} A)_{i j} A_{i j}$.

Lemma 2.1. We have

$$
\operatorname{det} D^{2} u=\frac{1}{n}\left(\operatorname{cof} D^{2} u\right): D^{2} u=\frac{1}{n} \operatorname{div}\left(\left(\operatorname{cof} D^{2} u\right) D u\right) .
$$

And for $F(u)=\operatorname{det} D^{2} u$ we have

$$
F^{\prime}(u)(w)=\left(\operatorname{cof} D^{2} u\right): D^{2} w=\operatorname{div}\left(\left(\operatorname{cof} D^{2} u\right) D w\right),
$$

for $u, w$ sufficiently smooth. 
Proof. Note that for any $n \times n$ matrix $A$, $\operatorname{det} A=(\operatorname{cof} A): A / n$, where $\operatorname{cof} A$ is the matrix of cofactors of $A$. This follows from the row expansion definition of the determinant.

For any sufficiently smooth matrix field $A$ and vector field $v, \operatorname{div} A^{T} v=(\operatorname{div} A) \cdot v+A$ : $D v$. Here the divergence of a matrix field is the divergence operator applied rowwise. If we put $v=D u$, then $\operatorname{det} D^{2} u=\left(\operatorname{cof} D^{2} u\right):\left(D^{2} u\right) / n=(\operatorname{cof} D v):(D v) / n$ and $\operatorname{div}(\operatorname{cof} D v)^{T} v=\operatorname{div}(\operatorname{cof} D v) \cdot v+(\operatorname{cof} D v): D v$. But $\operatorname{div} \operatorname{cof} D v=0$, c.f. for example [17] p. 440. Hence since $D^{2} u$ and cof $D^{2} u$ are symmetric matrices (2.5) follows. The assertion about the Fréchet derivative of $F$ follows from the definition of the determinant as a multilinear map (e.g (2.5) ) and the definition of matrix of cofactors. See also [17] p. 440.

Using the divergence form of the determinant (2.5) and integration by parts, one obtains the variational formulation of $(1.1)$ given by: find $u \in W^{2, n}(\Omega), u=g$ on $\partial \Omega$ such that

$$
-\frac{1}{n} \int_{\Omega}\left(\operatorname{cof} D^{2} u\right) D u \cdot D w d x=\int_{\Omega} f w d x, \quad \forall w \in W^{2, n}(\Omega) \cap H_{0}^{1}(\Omega) .
$$

We show that for $u \in W^{2, n}(\Omega),(2.6)$ is well defined.

Case $n=2$. For $n=2$, each entry of cof $D^{2} u$ consists of a second derivative $\partial^{2} u /\left(\partial x_{i} \partial x_{j}\right), i, j=1, \ldots, n$. By Hölder's inequality,

$$
\left|\int_{\Omega}\left(\operatorname{cof} D^{2} u\right) D u \cdot D w d x\right| \leq C|| D^{2} u\left\|_{0,2}|| D u\right\|_{0,4}\|D w\|_{0,4}
$$

Next for $u \in H^{2}(\Omega), \partial u / \partial x_{i} \in H^{1}(\Omega), i=1, \ldots, n$ and by Sobolev embedding, i.e. the embedding of $H^{1}(\Omega)$ in $L^{q}(\Omega)$ for $q \geq 1$ when $n=2$, the right hand side above is bounded by $C\left\|D^{2} u\right\|_{L^{2}(\Omega)}|| u\left\|_{H^{2}(\Omega)}\right\| w \|_{H^{2}(\Omega)}$.

Case $n=3$. For $n=3$, each entry of cof $D^{2} u$ involves the product of two second order derivatives. We have by Hölder's inequality and Sobolev embedding, i.e. the embedding of $H^{1}(\Omega)$ in $L^{q}(\Omega)$ for $1 \leq q \leq 6$ when $n=3$,

$$
\begin{aligned}
\left|\int_{\Omega} \frac{\partial^{2} u}{\partial x_{1}^{2}} \frac{\partial^{2} u}{\partial x_{3}^{2}} \frac{\partial u}{\partial x_{1}} \frac{\partial w}{\partial x_{2}} d x\right| & \leq\left\|\frac{\partial^{2} u}{\partial x_{1}^{2}}\right\|_{0,3}\left\|\frac{\partial^{2} u}{\partial x_{3}^{2}}\right\|_{0,3}\left\|\frac{\partial u}{\partial x_{1}}\right\|_{0,6}\left\|\frac{\partial w}{\partial x_{2}}\right\|_{0,6} \\
& \leq\|u\|_{2,3}^{2}\|u\|_{2}\|w\|_{2} .
\end{aligned}
$$

We conclude that for $n=3$,

$$
\left|\int_{\Omega}\left(\operatorname{cof} D^{2} u\right) D u \cdot D w d x\right| \leq C\|u\|_{2,3}^{2}\|u\|_{2}\|w\|_{2} .
$$

In summary for $n=2,3$, we may write

$$
\left|\int_{\Omega}\left(\operatorname{cof} D^{2} u\right) D u \cdot D w d x\right| \leq C\|u\|_{2, n}^{n-1}\|u\|_{2}\|w\|_{2} .
$$

Put $V=W^{2, n}(\Omega)$ and $V_{0}=W^{2, n}(\Omega) \cap H_{0}^{1}(\Omega)$. Note that $V^{h}$ given by (2.1) satisfies $V^{h} \subset W^{2, n}(\Omega)$. Let $V_{0}^{h}=V^{h} \cap H_{0}^{1}(\Omega)$ and furthermore let $g_{h}$ be the interpolant in $V^{h}$ of a smooth extension of $g$. 
We have the following conforming discretization of $(2.6)$ : find $u_{h} \in V^{h}, u_{h}=g_{h}$ on $\partial \Omega$ such that

$$
-\frac{1}{n} \int_{\Omega}\left(\operatorname{cof} D^{2} u_{h}\right) D u_{h} \cdot D w_{h} d x=\int_{\Omega} f w_{h} d x, \quad \forall w_{h} \in V_{0}^{h} .
$$

We now present a number of preliminary results.

2.2. Preliminary results. We first prove that when (1.1) has a smooth strictly convex solution, (2.8) has a unique local solution and we give error estimates. After introducing tools for computations with determinants, we show that a finite element function sufficiently close to a strictly convex finite element function is also strictly convex. It follows that the solution $u_{h}$ of $(2.8)$ is strictly convex when (1.1) has a smooth strictly convex solution.

Theorem 2.2. Let $3 \leq l \leq d$ and assume that $u \in W^{l+1, \infty}(\Omega)$ is a strictly convex function, that $\Omega$ is convex with a polygonal boundary and that the spaces $V^{h}$ have the optimal approximation property (2.2) and satisfy the inverse estimates (2.4). Then the problem (2.8) has a unique solution $u_{h}$ for $h$ sufficiently small and we have the error estimates

$$
\begin{aligned}
& \left\|u-u_{h}\right\|_{2} \leq C h^{l-1} \\
& \left\|u-u_{h}\right\|_{1} \leq C h^{l} \\
& \left\|u-u_{h}\right\|_{0} \leq C h^{l+1}+C\left(h^{l-1-\frac{n}{2}}+C\right)^{n-2} h^{2 l-1-\frac{n}{2}},
\end{aligned}
$$

with a constant $C$ which depends on $u$ but is independent of $h$.

Proof. The $H^{1}$ error estimate is given in [9], Theorems 5.1 and 8.7. See also [10]. The $H^{2}$ error estimate follows from an inverse estimate. For the proof of the $L^{2}$ error estimate, the proof in [22] can be adapted. The results of [12, 13] also give the error estimates in the theorem. They were given with the interior penalty formulation but the variational problems discussed there reduce to the one considered in this paper for $C^{1}$ finite element spaces. For another proof of the $H^{1}$ error estimate, we refer to [3].

Next we give some preliminary results which are essential for computations with terms involving the determinant.

We first recall the Mean Value Theorem for Banach spaces. Let $E$ and $F$ be Banach spaces and let us denote by $L(E, F)$ the space of continuous linear mappings from $E$ to $F$. Let also $X$ be an open subset of $E$ and let $F: X \rightarrow F$ be a differentiable map. If $F^{\prime}: X \rightarrow L(E, F)$ is continuous, $F$ is said to be of class $C^{1}$ and for all $a, x \in X$, we have

$$
F(x)=F(a)+\int_{0}^{1} F^{\prime}[(1-t) a+t x](x-a) d t .
$$

Lemma 2.3. For $n=2$ and $n=3$, and two matrix fields $\eta$ and $\tau$

$$
\|\operatorname{cof}(\eta)-\operatorname{cof}(\tau)\|_{\infty} \leq(n-1)^{2}\left(\|\eta\|_{\infty}+\|\tau\|_{\infty}\right)^{n-2}\|\eta-\tau\|_{\infty}
$$


Proof. For $n=2$, we have $\operatorname{cof}(\eta)-\operatorname{cof}(\tau)=\operatorname{cof}(\eta-\tau)$ from which the result follows. For $n=3$ we use the Mean Value Theorem. It is enough to estimate the first entry of $\operatorname{cof}(\eta)-\operatorname{cof}(\tau)$ which is equal to

$$
\begin{gathered}
\operatorname{det}\left(\begin{array}{ll}
\eta_{22} & \eta_{23} \\
\eta_{32} & \eta_{33}
\end{array}\right)-\operatorname{det}\left(\begin{array}{ll}
\tau_{22} & \tau_{23} \\
\tau_{32} & \tau_{33}
\end{array}\right) \\
=\operatorname{cof}\left(t\left(\begin{array}{ll}
\eta_{22} & \eta_{23} \\
\eta_{32} & \eta_{33}
\end{array}\right)+(1-t)\left(\begin{array}{ll}
\tau_{22} & \tau_{23} \\
\tau_{32} & \tau_{33}
\end{array}\right)\right): \\
\left(\begin{array}{ll}
\eta_{22}-\tau_{22} & \eta_{23}-\tau_{23} \\
\eta_{32}-\tau_{32} & \eta_{33}-\tau_{33}
\end{array}\right),
\end{gathered}
$$

for some $t \in[0,1]$. The result then follows.

Lemma 2.4. Let $v, w \in W^{2, n}(\Omega), n=2,3$ and $\psi \in H_{0}^{1}(\Omega) \cap H^{2}(\Omega)$, then

$$
\begin{array}{r}
\int_{\Omega}\left(\operatorname{det} D^{2} v-\operatorname{det} D^{2} w\right) \psi d x=-\int_{0}^{1}\left\{\int _ { \Omega } \left(\left(\operatorname{cof}\left[(1-t) D^{2} w+t D^{2} v\right)\right]\right.\right. \\
(D v-D w)) \cdot D \psi d x\} d t
\end{array}
$$

and if in addition $v, w \in W^{2, n}(\Omega) \cap W^{2, \infty}(\Omega)$

$$
\left|\int_{\Omega}\left(\operatorname{det} D^{2} v-\operatorname{det} D^{2} w\right) \psi d x\right| \leq n\left(|v|_{2, \infty}+|w|_{2, \infty}\right)^{n-1}|v-w|_{1}|\psi|_{1},
$$

and

$$
\begin{array}{r}
\left|\int_{\Omega}\left[\left(\operatorname{cof} D^{2} v-\operatorname{cof} D^{2} w\right) D(v-w)\right] \cdot D \psi d x\right| \leq n(n-1)^{2}\left(|v|_{2, \infty}\right. \\
\left.+|w|_{2, \infty}\right)^{n-2}|v-w|_{2, \infty}|v-w|_{1}|\psi|_{1} .
\end{array}
$$

Proof. We first note that for a matrix field $A$ and vector fields $b, c$, we have $(A b) \cdot c=$ $\sum_{i=1}^{n}(A b)_{i} c_{i}=\sum_{i, j=1}^{n} A_{i j} b_{j} c_{i}$. Thus by Cauchy-Schwarz inequality,

$$
\begin{aligned}
\int_{\Omega}(A b) \cdot c & \leq\|A\|_{\infty} \sum_{i, j=1}^{n} \int_{\Omega}\left|b_{j} c_{i}\right| \leq\|A\|_{\infty} \sum_{i, j=1}^{n}\left\|b_{j}\right\|_{0}\left\|c_{i}\right\|_{0} \\
& =\|A\|_{\infty}\left(\sum_{i=1}^{n}\left\|c_{i}\right\|_{0}\right)\left(\sum_{j=1}^{n}\left\|b_{j}\right\|_{0}\right) \\
& \leq n\|A\|_{\infty}\left(\sum_{i=1}^{n}\left\|c_{i}\right\|_{0}^{2}\right)^{\frac{1}{2}}\left(\sum_{j=1}^{n}\left\|b_{j}\right\|_{0}^{2}\right)^{\frac{1}{2}} .
\end{aligned}
$$

It follows that for $L^{\infty}$ valued matrix fields $A, B$ and $v, w \in W^{2, n}(\Omega), n=2,3$, by Cauchy-Schwarz inequality

$$
\left|\int_{\Omega}[(\operatorname{cof} A-\operatorname{cof} B) D v] D w d x\right| \leq n|| \operatorname{cof} A-\left.\operatorname{cof} B\left|\|_{\infty}\right| v\right|_{1}|w|_{1} .
$$

Next, let $F: C^{\infty}(\Omega) \rightarrow C^{\infty}(\Omega)$ denote the mapping $v \mapsto \operatorname{det} D^{2} v$. Then $F$ is differentiable with

$$
F^{\prime}[u](v)=\left(\operatorname{cof} D^{2} u\right): D^{2} v=\operatorname{div}\left(\left(\operatorname{cof} D^{2} u\right) D v\right) .
$$


Since $v \mapsto F^{\prime}[v]$ is linear, $F$ is of class $C^{1}$ and by the Mean Value Theorem

$$
F(v)-F(w)=\int_{0}^{1} \operatorname{div}\left(\left(\operatorname{cof}(1-t) D^{2} w+t D^{2} v\right)(D v-D w)\right) d t .
$$

It follows that for $\psi \in \mathcal{D}(\Omega)$, and $v, w \in C^{\infty}(\Omega) \cap W^{2, \infty}(\Omega)$,

$$
\int_{\Omega}\left(\operatorname{det} D^{2} v-\operatorname{det} D^{2} w\right) \psi d x=\int_{\Omega}\left\{\int_{0}^{1} \operatorname{div}\left(\left(\operatorname{cof}(1-t) D^{2} w+t D^{2} v\right)(D v-D w)\right) d t\right\} \psi d x \text {. }
$$

By Fubini's theorem,

$$
\begin{aligned}
\int_{\Omega}\left(\operatorname{det} D^{2} v-\operatorname{det} D^{2} w\right) \psi d x & =\int_{0}^{1}\left\{\int_{\Omega} \operatorname{div}\left(\left(\operatorname{cof}(1-t) D^{2} w+t D^{2} v\right)(D v-D w)\right) \psi d x\right\} d t \\
= & -\int_{0}^{1}\left\{\int_{\Omega}\left[\left(\operatorname{cof}(1-t) D^{2} w+t D^{2} v\right)(D v-D w)\right] \cdot D \psi d x\right\} d t .
\end{aligned}
$$

Applying (2.11), we obtain

$$
\begin{aligned}
\left|\int_{\Omega}\left(\operatorname{det} D^{2} v-\operatorname{det} D^{2} w\right) \psi d x\right| & \leq n \int_{0}^{1}\left\|\operatorname{cof}(1-t) D^{2} w+t D^{2} v\right\|_{\infty}|v-w|_{1}|\psi|_{1} d t \\
& \leq n \int_{0}^{1}\left\|(1-t) D^{2} w+t D^{2} v\right\|_{\infty}^{n-1}|v-w|_{1}|\psi|_{1} d t \\
& \leq n\left(|v|_{2, \infty}+|w|_{2, \infty}\right)^{n-1}|v-w|_{1}|\psi|_{1} .
\end{aligned}
$$

We have therefore obtained 2.9 for $v, w \in C^{\infty}(\Omega) \cap W^{2, \infty}(\Omega)$ and $\psi \in \mathcal{D}(\Omega)$. We recall that $\mathcal{D}(\Omega)$ is dense in $H_{0}^{1}(\Omega) \cap H^{2}(\Omega)$ and $C^{\infty}(\Omega) \cap W^{2, \infty}(\Omega)$ is dense in $W^{2, \infty}(\Omega)$. We then obtain $(2.9)$ by a density argument.

Inequality (2.10) is a direct consequence of (2.11) and Lemma 2.3 .

Let $\lambda_{1}(A)$ and $\lambda_{n}(A)$ denote the smallest and largest eigenvalues of a symmetric matrix $A$. Since $\operatorname{det} D^{2} u \geq f \geq c_{0}>0$ and $u$ is smooth and convex, there exist constants $m^{\prime}, M^{\prime}>0$, independent of $h$

$$
m^{\prime} \leq \lambda_{1}\left(D^{2} u(x)\right) \leq \lambda_{n}\left(D^{2} u(x)\right) \leq M^{\prime}, \forall x \in \Omega .
$$

It follows from [24] Theorem 1 and Remark 2 p. 39 that for two symmetric $n \times n$ matrices $A$ and $B$,

$$
\left|\lambda_{k}(A)-\lambda_{k}(B)\right| \leq n \max _{i, j}\left|A_{i j}-B_{i j}\right|, k=1, \ldots, n .
$$

It follows that for $u, v \in W^{2, \infty}(\Omega)$,

$$
\begin{aligned}
\left|\lambda_{1}\left(D^{2} u(x)\right)-\lambda_{1}\left(D^{2} v(x)\right)\right| & \leq n|u-v|_{2, \infty} \\
\left|\lambda_{n}\left(D^{2} u(x)\right)-\lambda_{n}\left(D^{2} v(x)\right)\right| & \leq n|u-v|_{2, \infty} .
\end{aligned}
$$

By (2.4) we have for $v \in V^{h}$

$$
|v|_{2, \infty} \leq C_{0} h^{-1-\frac{n}{2}}\|v\|_{1}
$$

Let $\delta>0$ such that

$$
\delta<\min \left\{1, \frac{m^{\prime}}{2 n C_{0}}\right\}
$$


Lemma 2.5. For $h$ sufficiently small and for all $v_{h} \in V^{h}$ with $\left\|v_{h}-Q_{h} u\right\|_{1}<$ $\delta h^{1+n / 2} / 2, D^{2}\left(\left.v_{h}\right|_{K}\right)$ is positive definite with

$$
\frac{m^{\prime}}{2} \leq \lambda_{1} D^{2}\left(\left.v_{h}\right|_{K}\right) \leq \lambda_{n} D^{2}\left(\left.v_{h}\right|_{K}\right) \leq \frac{3 M^{\prime}}{2}
$$

where $m^{\prime}$ and $M^{\prime}$ are the constants of Assumption (2.12). It follows that $v_{h}$ is convex.

Proof. For $v \in W^{2, \infty}(\Omega),|v-u|_{2, \infty} \leq \delta C_{0}$ and 2.16 imply

$$
\left|\lambda_{1}\left(D^{2} v(x)\right)-\lambda_{1}\left(D^{2} u(x)\right)\right| \leq n|v-u|_{2, \infty} \leq n \delta C_{0} \leq \frac{m^{\prime}}{2} \text { a.e. in } \Omega,
$$

since $\delta<m^{\prime} /\left(2 n C_{0}\right.$. By Assumption 2.12) $\lambda_{1}\left(D^{2} u(x)\right) \geq m^{\prime}$, and thus $\lambda_{1}\left(D^{2} v(x)\right) \geq$ $\lambda_{1}\left(D^{2} u(x)\right)-m^{\prime} / 2 \geq m^{\prime} / 2$ a.e. in $\Omega$. We conclude that for $|v-u|_{2, \infty} \leq \delta C_{0}$, $\lambda_{1}\left(D^{2} v(x)\right)>m^{\prime} / 2$, a.e. in $\Omega$.

Now, by (2.2), $\left|u-Q_{h} u\right|_{2, \infty} \leq C h^{d-1}|u|_{d+1, \infty}$. So for $h$ sufficiently small, $\left|u-Q_{h} u\right|_{2, \infty} \leq$ $\delta C_{0} / 2$. Moreover by 2.4 and the assumption of the lemma

$$
\left|v_{h}-Q_{h} u\right|_{2, \infty} \leq C_{0} h^{-1-\frac{n}{2}}\left\|v_{h}-Q_{h} u\right\|_{1} \leq \frac{\delta C_{0}}{2} .
$$

Therefore $\left|v_{h}-u\right|_{2, \infty} \leq \delta C_{0}$ as well and it follows that $\lambda_{1}\left(D^{2} v_{h}(x)\right)>m^{\prime} / 2$, a.e. in $\Omega$ as claimed.

Since $m^{\prime} \leq M^{\prime}$, we also have $\left|\lambda_{n}\left(D^{2} v_{h}(x)\right)-\lambda_{n}\left(D^{2} u(x)\right)\right| \leq M^{\prime} / 2$ a.e. in $\Omega$. Thus $\lambda_{n}\left(D^{2} v_{h}(x)\right) \leq \lambda_{n}\left(D^{2} u(x)\right)+M^{\prime} / 2 \leq 3 M^{\prime} / 2$.

Since $v_{h}$ is piecewise convex and $C^{1}, v_{h}$ is convex [27] Lemma 1. This concludes the proof.

Put

$$
X^{h}=\left\{v_{h} \in V^{h}, v_{h}=g_{h} \text { on } \partial \Omega,\left\|v_{h}-Q_{h} u\right\|_{1}<\frac{\delta h^{1+\frac{n}{2}}}{4}\right\} .
$$

By Lemma 2.5, for $h$ sufficiently small and $v_{h} \in X^{h},\left\|v_{h}-Q_{h} u\right\|_{1}<\delta h^{1+n / 2} / 2$ and hence $v_{h}$ is convex with smallest eigenvalue bounded a.e. below by $m^{\prime} / 2$ and above by $3 M^{\prime} / 2$.

As a consequence of Assumption 2.12 we have

Lemma 2.6. For $h$ sufficiently small and all $v_{h} \in X_{h}$

$$
m \leq \lambda_{1}\left(\operatorname{cof} D^{2} v_{h}(x)\right) \leq \lambda_{n}\left(\operatorname{cof} D^{2} v_{h}(x)\right) \leq M, \forall x \in K, K \in \mathcal{T}_{h},
$$

with $m=\left(m^{\prime}\right)^{n} / M^{\prime}$ and $M=\left(M^{\prime}\right)^{n} / m$.

It follows that for $w \in H^{1}(K)$

$$
m|w|_{1, K}^{2} \leq \int_{K}\left[\left(\operatorname{cof} D^{2} v_{h}(x)\right) D w(x)\right] \cdot D w(x) d x \leq M|w|_{1, K}^{2} .
$$

Proof. We first note that by Lemma 2.5, there exist constants $m, M>0$ such that $m \leq \lambda_{1}\left(\operatorname{cof} D^{2} v_{h}(x)\right) \leq \lambda_{n}\left(\operatorname{cof} D^{2} v_{h}(x)\right) \leq M$ a.e. in $\Omega$ for $v_{h} \in X_{h}$. To prove this, recall that for an invertible matrix $A$, $\operatorname{cof} A=(\operatorname{det} A)\left(A^{-1}\right)^{T}$. Since a matrix and its transpose have the same set of eigenvalues, the eigenvalues of $\operatorname{cof} A$ are of the form $\operatorname{det} A / \lambda_{i}$ where $\lambda_{i}, i=1, \ldots, n$ is an eigenvalue of $A$. Applying this observation to 
$A=D^{2} u(x)$ and using Lemma 2.5, we obtain that the eigenvalues of cof $D^{2} v_{h}(x)$ are a.e. uniformly bounded below by $m=\left(m^{\prime}\right)^{n} / M^{\prime}$ and above by $M=\left(M^{\prime}\right)^{n} / m$.

Since $\lambda_{1}\left(D^{2} v_{h}(x)\right)$ and $\lambda_{n}\left(D^{2} v_{h}(x)\right)$ are the minimum and maximum respectively of the Rayleigh quotient $\left[\left(\operatorname{cof} D^{2} v_{h}(x)\right) z\right] \cdot z /\|z\|^{2}$, where $\|z\|$ denotes the standard Euclidean norm in $\mathbb{R}^{n}$, we have

$$
m\|z\|^{2} \leq\left[\left(\operatorname{cof} D^{2} v_{h}(x)\right) z\right] \cdot z \leq M\|z\|^{2}, z \in \mathbb{R}^{n} .
$$

This implies

$$
m|w|_{1, K}^{2} \leq \int_{K}\left[\left(\operatorname{cof} D^{2} v_{h}(x)\right) D w(x)\right] \cdot D w(x) d x \leq M|w|_{1, K}^{2}, w \in H^{1}(K) .
$$

Remark 2.7. As a consequence of Lemma 2.5 and Theorem 2.2. for $h$ sufficiently small, the solution $u_{h}$ of 2.8 is in $X^{h}$ and hence convex.

Let $\left(V_{0}^{h}\right)^{\prime}$ denote the dual space of $V_{0}^{h}$ with $V_{0}^{h}$ equipped with $\|.\|_{1}$. We consider the mapping $F_{h}: V^{h} \rightarrow\left(V_{0}^{h}\right)^{\prime}$ defined by

$$
\left\langle F_{h}\left(v_{h}\right), \psi_{h}\right\rangle=\int_{\Omega}\left(\operatorname{det} D^{2} v_{h}\right) \psi_{h} d x, v_{h} \in V^{h}, \psi_{h} \in V_{0}^{h}
$$

and recall that $f>0$ is continuous. Since $\Omega$ is bounded, by $L^{2}$ duality, $V^{h} \subset\left(V_{0}^{h}\right)^{\prime}$. We use the notation $\|$.$\| for the operator norm of an element of a dual space. With this$ notation, (2.6) can be written $\operatorname{det} D^{2} u=f$ in $V_{0}^{\prime}$ and (2.8) can be written $F_{h}\left(u_{h}\right)=f$ in $\left(V_{0}^{h}\right)^{\prime}$.

Let $C_{1}$ denote the constant in the Poincare's inequality, i.e. $\|p\|_{1} \leq C_{1}|p|_{1}, p \in H_{0}^{1}(\Omega)$. Without loss of generality, we may assume that $C_{1} \leq 1$, for example by assuming that the domain $\Omega$ is contained in a cube of side length at most 1, [11] p.30.

Note that by Lemma 2.1 and integration by parts,

$$
\left\langle F_{h}^{\prime}\left(v_{h}\right)(p), p\right\rangle=-\int_{\Omega}\left[\left(\operatorname{cof} D^{2} v_{h}\right) D p\right] \cdot D p d x, p \in H_{0}^{1}(\Omega) .
$$

Thus by Lemma 2.6 and $h$ sufficiently small

$$
-M\|p\|_{1}^{2} \leq\left\langle F_{h}^{\prime}\left(v_{h}\right)(p), p\right\rangle \leq-\frac{m}{C_{1}^{2}}\|p\|_{1}^{2}, p \in H_{0}^{1}(\Omega),
$$

for $v_{h} \in X^{h}$. We have

Lemma 2.8. The following properties hold for h sufficiently small.

Discrete coercivity:

$$
\left\|F_{h}^{\prime}\left(v_{h}\right)(p)\right\| \geq \frac{m}{C_{1}^{2}}\|p\|_{1}, \forall p \in V_{0}^{h} \text { and } v_{h} \in X^{h} .
$$

Generalized Lipschitz continuity:

$$
\left\|F_{h}^{\prime}\left(v_{h}\right)(\psi)-F_{h}^{\prime}\left(w_{h}\right)(\psi)\right\| \leq C_{2} h^{-1-\frac{n}{2}}\left\|v_{h}-w_{h}\right\|_{1}\|\psi\|_{1},
$$

for $v_{h}, w_{h} \in X^{h}$ and $\psi \in V^{h}, \eta \in V_{0}^{h}$ and with $C_{2}=C\|u\|_{2, \infty}$. 
Proof. By (2.19) $\left\|F_{h}^{\prime}(v)(p)\right\|=\sup _{\psi \neq 0}\left|\left\langle F_{h}^{\prime}(v)(p), \psi\right\rangle\right| /\|\psi\|_{1} \geq m / C_{1}^{2}\|p\|_{1}$, which proves the discrete coercivity condition.

For $v_{h}, w_{h} \in X^{h}, \psi \in V^{h}, \eta \in V_{0}^{h}$, we have

$$
\begin{aligned}
\left\langle F_{h}^{\prime}\left(v_{h}\right)(\psi), \eta\right\rangle-\left\langle F_{h}^{\prime}\left(w_{h}\right)(\psi), \eta\right\rangle=\int_{\Omega}\left(\operatorname{div}\left(\operatorname{cof} D^{2} v_{h}\right) D \psi\right) \eta d x & -\int_{\Omega}\left(\operatorname{div}\left(\operatorname{cof} D^{2} w_{h}\right) D \psi\right) \eta d x \\
= & -\int_{\Omega}\left[\left(\operatorname{cof} D^{2} v_{h}\right) D \psi\right] \cdot D \eta d x \\
+\int_{\Omega}\left[\left(\operatorname{cof} D^{2} w_{h}\right) D \psi\right] \cdot D \eta d x & \\
= & \int_{\Omega}\left[\left(\operatorname{cof} D^{2} w_{h}-\operatorname{cof} D^{2} v_{h}\right) D \psi\right] \cdot D \eta d x
\end{aligned}
$$

By (2.10) and an inverse estimate

$$
\begin{gathered}
\left|\left\langle F_{h}^{\prime}\left(v_{h}\right)(\psi), \eta\right\rangle-\left\langle F_{h}^{\prime}\left(w_{h}\right)(\psi), \eta\right\rangle\right| \leq n(n-1)^{2}\left(\left\|v_{h}\right\|_{2, \infty}\right. \\
\left.+\left\|w_{h}\right\|_{2, \infty}\right)^{n-2}\left\|v_{h}-w_{h}\right\|_{2, \infty}\|\psi\|_{1}\|\eta\|_{1} \\
\left\|F_{h}^{\prime}\left(v_{h}\right)(\psi)-F_{h}^{\prime}\left(w_{h}\right)(\psi)\right\| \leq n(n-1)^{2}\left(\left\|v_{h}\right\|_{2, \infty}\right. \\
\left.+\left\|w_{h}\right\|_{2, \infty}\right)^{n-2}\left\|v_{h}-w_{h}\right\|_{2, \infty}\|\psi\|_{1} \\
\leq n(n-1)^{2} C_{0}\left(\left\|v_{h}\right\|_{2, \infty}+\left\|w_{h}\right\|_{2, \infty}\right)^{n-2} \\
\left\|v_{h}-w_{h}\right\|\left\|_{1}\right\| \psi \|_{1} .
\end{gathered}
$$

In the case $n=3$ we have by an inverse estimate, the definition of $X^{h}$ and the assumption on $\delta(2.16)$

$$
\begin{aligned}
\left\|v_{h}\right\|_{2, \infty}+\left\|w_{h}\right\|_{2, \infty} & \leq\left\|v_{h}-Q_{h} u\right\|_{2, \infty}+\left\|w_{h}-Q_{h} u\right\|_{2, \infty}+2\left\|Q_{h} u\right\|_{2, \infty} \\
& \leq C_{0} h^{-1-\frac{3}{2}}\left(\left\|v_{h}-Q_{h} u\right\|_{1}+\left\|w_{h}-Q_{h} u\right\|_{1}\right) \\
& +C\|u\|_{2, \infty} \\
& \leq \frac{C_{0} \delta}{2}+C\|u\|_{2, \infty} \\
& \leq \frac{C_{0}}{2}+C\|u\|_{2, \infty} .
\end{aligned}
$$

We conclude that 2.20 holds.

We define

$$
Y^{h}=\left\{v_{h} \in V^{h}, v_{h}=g_{h} \text { on } \partial \Omega,\left\|v_{h}-u_{h}\right\|_{1}<\frac{\delta h^{1+\frac{n}{2}}}{4}\right\} .
$$

Remark 2.9. For $v_{h} \in Y^{h},\left\|v_{h}-Q_{h} u\right\|_{1} \leq\left\|v_{h}-u_{h}\right\|_{1}+\left\|Q_{h} u-u_{h}\right\|_{1}<\delta h^{1+n / 2} / 2$ and hence $Y^{h} \subset X^{h}$.

We will make the abuse of notation of denoting by both $u_{k}$ the solution of the iterative methods at both the continuous and discrete level. In the remainder of this paper, only discrete solutions are considered. This alleviates the notation. 
Finally we note that we have the freedom to choose $\delta$ given by 2.16 smaller. Indeed this will be necessary for the convergence of the pseudo transient continuation methods.

We make the assumption that (2.8) has a unique strictly convex solution. Recall from Remark 2.7 that this holds for example when (1.1) has a smooth strictly convex solution and for $h$ sufficiently small.

\section{Convergence of the Pseudo transient Continuation methods}

We now prove the convergence of the iterative methods 1.2 . The discretization of (1.2) depends on the choice of $L$ : Given $\nu>0$ and a suitable initial guess, find

$u_{k+1} \in V^{h}, u_{k+1}=g_{h}$ on $\partial \Omega$ such that we have for all $\psi_{h} \in V_{0}^{h}$, when $L$ is the Laplace operator

$$
\begin{aligned}
-\nu \int_{\Omega}\left(D u_{k+1}-D u_{k}\right) \cdot D \psi_{h} d x+\left\langleF _ { h } ^ { \prime } ( u _ { k } ) \left( u_{k+1}\right.\right. & \left.\left.-u_{k}\right), \psi_{h}\right\rangle \\
& =\left\langle-\left(F_{h}\left(u_{k}\right)-f\right), \psi_{h}\right\rangle,
\end{aligned}
$$

and when $L$ is the negative of the identity,

$$
\begin{aligned}
-\nu \int_{\Omega}\left(u_{k+1}-u_{k}\right) \psi_{h} d x+\left\langle F_{h}^{\prime}\left(u_{k}\right)\right. & \left.\left(u_{k+1}-u_{k}\right), \psi_{h}\right\rangle \\
= & \left\langle-\left(F_{h}\left(u_{k}\right)-f\right), \psi_{h}\right\rangle .
\end{aligned}
$$

We define

$$
C_{h}=\frac{m}{C_{1}^{2}}+\frac{\nu h^{2}}{C_{0}^{2}}
$$

and require $h<\min \left\{\sqrt{2} C_{0}, C_{0} / C_{1}\right\}$. Thus since $h^{2}<2 C_{0}^{2}$ we have $2-h^{2} / C_{0}^{2}>0$ and if we require

$$
0<\nu<\frac{m}{C_{1}^{2}\left(2-\frac{h^{2}}{C_{0}^{2}}\right)},
$$

we have

$$
0<\nu<\frac{C_{h}}{2}
$$

We now require that

$$
\delta<\min \left\{1, \frac{m^{\prime}}{2 n C_{0}}, \frac{2 C_{h}}{C_{2}}\right\} .
$$

Theorem 3.1. Let $\Omega$ be convex with a Lipschitz continuous boundary and assume that the spaces $V^{h}=S_{d}^{1}(\mathcal{T})$ have the optimal approximation property (2.2) and satisfy the inverse estimates (2.4). A sequence defined by either (3.1) or (3.2) with a suitable initial guess and for $\nu, h$ sufficiently small converges to the unique strictly convex solution of (2.8). Moreover the convergence rate is linear. 
Proof. Define $\mathcal{M}_{i}: V_{0}^{h} \rightarrow\left(V_{0}^{h}\right)^{\prime}, i=1,2$ for $v, \psi_{h} \in V_{0}^{h}$ by

$$
\left\langle\mathcal{M}_{1}(v), \psi_{h}\right\rangle=\int_{\Omega} D v \cdot D \psi_{h} d x,\left\langle\mathcal{M}_{2}(v), \psi_{h}\right\rangle=\int_{\Omega} v \psi_{h} d x
$$

We note that

$$
\left\|\mathcal{M}_{i}(v)\right\| \leq\|v\|_{1}, v \in V_{0}^{h}, i=1,2 .
$$

Next, for $p \in V_{0}^{h}$, by 2.19 and Poincare's inequality we have

$$
-(M+\nu)\|p\|_{1}^{2} \leq\left\langle F_{h}^{\prime}\left(v_{h}\right)(p), p\right\rangle-\nu|p|_{1}^{2} \leq-\frac{1}{C_{1}^{2}}(m+\nu)\|p\|_{1}^{2}
$$

Thus

$$
\left.\left.\left|\left\langle F_{h}^{\prime}\left(v_{h}\right)(p), p\right\rangle-\nu\right| p\right|_{1} ^{2}\left|=-\left\langle F_{h}^{\prime}\left(v_{h}\right)(p), p\right\rangle+\nu\right| p\right|_{1} ^{2} \geq \frac{1}{C_{1}^{2}}(m+\nu)\|p\|_{1}^{2}
$$

We have

$$
\begin{aligned}
\left\|-\nu \mathcal{M}_{1}(p)+F_{h}^{\prime}\left(v_{h}\right)(p)\right\| & =\sup _{\psi_{h} \neq 0} \frac{\left|-\nu \mathcal{M}_{1}(p)\left(\psi_{h}\right)+F_{h}^{\prime}\left(v_{h}\right)(p)\left(\psi_{h}\right)\right|}{|| \psi_{h} \|_{1}} \\
& \geq \frac{\left|-\nu \mathcal{M}_{1}(p)(p)+F_{h}^{\prime}\left(v_{h}\right)(p)(p)\right|}{\|p\|_{1}} \\
& =\frac{\left.|-\nu| p\right|_{1} ^{2}+F_{h}^{\prime}\left(v_{h}\right)(p)(p) \mid}{\|p\|_{1}} \\
& \geq \frac{1}{C_{1}^{2}}(m+\nu)\|p\|_{1} .
\end{aligned}
$$

Similarly, since $C_{0}^{-2} h^{2}\|p\|_{1}^{2} \leq\|p\|_{0}^{2} \leq\|p\|_{1}^{2}$

$$
-(M+\nu)\|p\|_{1}^{2} \leq\left\langle F_{h}^{\prime}\left(v_{h}\right)(p), p\right\rangle-\nu\|p\|_{0}^{2} \leq-\left(\frac{m}{C_{1}^{2}}+\frac{\nu h^{2}}{C_{0}^{2}}\right)\|p\|_{1}^{2}
$$

And again

$$
\begin{aligned}
\left\|-\nu \mathcal{M}_{2}(p)+F_{h}^{\prime}\left(v_{h}\right)(p)\right\| & \geq \frac{\left|-\nu\|p\|_{0}^{2}+F_{h}^{\prime}\left(v_{h}\right)(p)(p)\right|}{\|p\|_{1}} \\
& \geq\left(\frac{m}{C_{1}^{2}}+\frac{\nu h^{2}}{C_{0}^{2}}\right)\|p\|_{1} .
\end{aligned}
$$

For $h$ sufficiently small, i.e. $h \leq C_{0} / C_{1}$, we have $1 / C_{1}^{2} \geq h^{2} / C_{0}^{2}$ and we therefore have

$$
\|p\|_{1} \leq \frac{1}{\frac{m}{C_{1}^{2}}+\frac{\nu h^{2}}{C_{0}^{2}}}\left\|-\nu \mathcal{M}_{i}(p)+F_{h}^{\prime}\left(v_{h}\right)(p)\right\|, p \in V_{0}^{h}, i=1,2 .
$$


We can now determine under which conditions when $u_{k} \in Y^{h}$ we have $u_{k+1} \in Y^{h}$ as well. Using (3.1), (3.2), $F_{h}\left(u_{h}\right)=f$ and the Mean Value Theorem,

$$
\begin{gathered}
-\nu \mathcal{M}_{i}\left(u_{k+1}-u_{h}\right)+F_{h}^{\prime}\left(u_{k}\right)\left(u_{k+1}-u_{h}\right)=-\nu \mathcal{M}_{i}\left(u_{k}-u_{h}\right)+F_{h}^{\prime}\left(u_{k}\right)\left(u_{k}-u_{h}\right) \\
-\left(F_{h}\left(u_{k}\right)-f\right) \\
=-\nu \mathcal{M}_{i}\left(u_{k}-u_{h}\right)+F_{h}^{\prime}\left(u_{k}\right)\left(u_{k}-u_{h}\right) \\
\quad-\int_{0}^{1} F_{h}^{\prime}\left(u_{h}+\theta\left(u_{k}-u_{h}\right)\right)\left(u_{k}-u_{h}\right) d \theta \\
=\int_{0}^{1}\left[F_{h}^{\prime}\left(u_{k}\right)-F_{h}^{\prime}\left(u_{h}+\theta\left(u_{k}-u_{h}\right)\right)\right]\left(u_{k}-u_{h}\right) \\
-\nu \mathcal{M}_{i}\left(u_{k}-u_{h}\right) d \theta,
\end{gathered}
$$

Using (3.3), (3.9), (3.7) and the generalized Lipschitz continuity property of $F_{h}^{\prime}$, we get

$$
C_{h}\left\|u_{k+1}-u_{h}\right\|_{1} \leq \nu\left\|u_{k}-u_{h}\right\|_{1}+C_{2} h^{-1-\frac{n}{2}}\left\|u_{k}-u_{h}\right\|_{1}^{2},
$$

and thus

$$
\left\|u_{k+1}-u_{h}\right\|_{1} \leq \frac{\nu}{C_{h}}\left\|u_{k}-u_{h}\right\|_{1}+\frac{C_{2} h^{-1-\frac{n}{2}}}{C_{h}}\left\|u_{k}-u_{h}\right\|_{1}^{2},
$$

By the definition of $Y^{h}$ and the choice of $\delta$, we have

$$
\frac{C_{2} h^{-1-\frac{n}{2}}}{C_{h}}\left\|u_{k}-u_{h}\right\|_{1} \leq \frac{\delta C_{2}}{4 C_{h}}<\frac{1}{2} .
$$

This gives by (3.5) and (3.10)

$$
\left\|u_{k+1}-u_{h}\right\|_{1}<\left\|u_{k}-u_{h}\right\|_{1}
$$

and we have proved that $u_{k+1} \in Y^{h}$ when $u_{k} \in Y^{h}$.

We now assume that $u_{0}$ is chosen in $Y^{h}$. We have for $i=1,2$

$$
\begin{aligned}
\left\langle F_{h}\left(u_{k+1}\right)-f, \psi_{h}\right\rangle= & \left\langle F_{h}\left(u_{k+1}\right)-F_{h}\left(u_{k}\right)+F_{h}\left(u_{k}\right)-f, \psi_{h}\right\rangle \\
= & \left\langle F_{h}\left(u_{k+1}\right)-F_{h}\left(u_{k}\right), \psi_{h}\right\rangle-\left\langle\nu \mathcal{M}_{i}\left(u_{k+1}-u_{k}\right), \psi_{h}\right\rangle \\
- & -\left\langle F_{h}^{\prime}\left(u_{k}\right)\left(u_{k+1}-u_{k}\right), \psi_{h}\right\rangle \\
=\left\langle\int_{0}^{1}\left[F_{h}^{\prime}\left(u_{k}+t\left(u_{k+1}-u_{k}\right)\right)-F_{h}^{\prime}\left(u_{k}\right)\right]\left(u_{k+1}-u_{k}\right) d t\right. & \quad-\left\langle\nu \mathcal{M}_{i}\left(u_{k+1}-u_{k}\right), \psi_{h}\right\rangle .
\end{aligned}
$$

We conclude from the general Lipschitz continuity property with $v_{h}=u_{k}$, an inverse estimate and (3.7),

$$
\left\|F_{h}\left(u_{k+1}\right)-f\right\| \leq C_{2} h^{-1-\frac{n}{2}}\left\|u_{k+1}-u_{k}\right\|_{1}^{2}+\nu\left\|u_{k+1}-u_{k}\right\|_{1} .
$$

Finally, by (3.9) and the definition of the iterative methods (3.1) and (3.2),

$$
\left\|u_{k+1}-u_{k}\right\|_{1} \leq \frac{1}{C_{h}}\left\|F_{h}\left(u_{k}\right)-f\right\| .
$$

We conclude that

$$
\left\|F_{h}\left(u_{k+1}\right)-f\right\| \leq c_{1}(h)\left\|F_{h}\left(u_{k}\right)-f\right\|^{2}+c_{0}(h)\left\|F_{h}\left(u_{k}\right)-f\right\|,
$$


for constants $c_{0}(h)=\nu / C_{h}$ and $c_{1}(h)$ which depends on $h$. By the assumption (3.5) on $\nu$, we have $c_{0}(h)<1 / 2$.

Let $q=\left\|F_{h}\left(u_{0}\right)-f\right\|$ and assume that $u_{0}$ is chosen so that $c_{1}(h) q<1-c_{0}(h)$. We then have

It follows that

$$
s \equiv c_{1}(h) q+c_{0}(h)<1
$$

$$
\left\|F_{h}\left(u_{1}\right)-f\right\| \leq c_{1}(h)\left\|F_{h}\left(u_{0}\right)-f\right\|^{2}+c_{0}(h)\left\|F_{h}\left(u_{0}\right)-f\right\|=s q,
$$

and since $s<1$,

$$
\begin{aligned}
\left\|F_{h}\left(u_{2}\right)-f\right\| & \leq c_{1}(h)\left\|F_{h}\left(u_{1}\right)-f\right\|^{2}+c_{0}(h)\left\|F_{h}\left(u_{1}\right)-f\right\| \\
& =\left\|F_{h}\left(u_{1}\right)-f\right\|\left(c_{1}(h)\left\|F_{h}\left(u_{1}\right)-f\right\|+c_{0}(h)\right) \\
& \leq\left\|F_{h}\left(u_{1}\right)-f\right\|\left(c_{1}(h) s q+c_{0}(h)\right) \\
& \leq\left\|F_{h}\left(u_{1}\right)-f\right\| s \leq s^{2} q .
\end{aligned}
$$

We conclude that $\left\|F_{h}\left(u_{k}\right)-f\right\| \leq s^{k} q$. Using $F_{h}\left(u_{h}\right)=f$ and the Mean Value Theorem

$$
\begin{aligned}
\left\langle F_{h}\left(u_{k}\right)-f, u_{k}-u_{h}\right\rangle & =\left\langle F_{h}\left(u_{k}\right)-F_{h}\left(u_{h}\right), u_{k}-u_{h}\right\rangle \\
& =\left\langle\int_{0}^{1} F_{h}^{\prime}\left(t u_{k}+(1-t) u_{h}\right)\left(u_{k}-u_{h}\right) d t, u_{k}-u_{h}\right\rangle \\
& =\left\langle\int_{0}^{1} F_{h}^{\prime}\left(t u_{k}+(1-t) u_{h}\right)\left(u_{k}-u_{h}\right), u_{k}-u_{h}\right\rangle d t
\end{aligned}
$$

Thus integrating 2.19 with respect to $t$ we obtain

$$
\begin{aligned}
\frac{m}{C_{1}^{2}}\left\|u_{k}-u_{h}\right\|_{1}^{2} & \leq\left|\int_{0}^{1}\left\langle F_{h}^{\prime}\left(t u_{k}+(1-t) u_{h}\right)\left(u_{k}-u_{h}\right), u_{k}-u_{h}\right\rangle d t\right| \\
& =\left|\left\langle F_{h}\left(u_{k}\right)-f, u_{k}-u_{h}\right\rangle\right| \\
& \leq\left\|F_{h}\left(u_{k}\right)-f\right\|\left\|u_{k}-u_{h}\right\|_{1} .
\end{aligned}
$$

We conclude that

$$
\left\|u_{k}-u_{h}\right\|_{1} \leq C\left\|F_{h}\left(u_{k}\right)-f\right\| \leq C q s^{k},
$$

from which the convergence follows. The convergence rate is given by (3.11).

Remark 3.2. The proof of convergence of the pseudo transient continuation methods also gives the convergence of Newton's method when $\nu=0$. In particular (3.10) gives the quadratic convergence rate of Newton's method when $\nu=0$. The quadratic convergence rate of Newton's method was also proved in a more general context in [9] where it is shown that the rate of convergence is independent of $h$, [9] Theorem 9.1. The independence of the rate in terms of the mesh size is known as mesh independence principle.

Remark 3.3. The introduction of the constant $C_{h}$ is motivated by our desire to have a unified analysis in (3.9) for both types of pseudo transient continuation methods. Since

$$
\|p\|_{1} \leq C\left\|-\nu \mathcal{M}_{1}(p)+F_{h}^{\prime}\left(v_{h}\right)(p)\right\|, p \in V_{0}^{h}
$$


we get for $\nu=0$ from 3.10

$$
\left\|u_{k+1}-u_{h}\right\|_{1} \leq C h^{-1-\frac{n}{2}}\left\|u_{k}-u_{h}\right\|_{1}^{2}
$$

for a constant $C$ independent of $h$.

Remark 3.4. The analysis above does not indicate whether (3.1) should be preferred over (3.2). We view (3.1) as a preconditioned version of (3.2). Moreover, the numerical results indicate that the use of the Laplacian preconditioner improves the convexity property of the numerical solution.

\section{Convergence of the time MARChing Methods}

We now turn to the proof of one of the main results of this paper, the convergence analysis of the iterative method $(1.3)$ for the Monge-Ampère equation.

Let $\nu=(M+m) / 2$ and define a mapping $T_{1}: Y^{h} \rightarrow\left(V_{0}^{h}\right)^{\prime}$ by

$$
\left\langle T_{1}\left(v_{h}\right), \psi_{h}\right\rangle=\int_{\Omega} D v_{h} \cdot D \psi_{h} d x+\frac{1}{\nu} \int_{\Omega}\left(\operatorname{det} D^{2} v_{h}-f\right) \psi_{h} d x
$$

for $v_{h} \in Y^{h}, \psi_{h} \in V_{0}^{h}$. The following lemma will make it possible to show that $T_{1}$ is a strict contraction.

Lemma 4.1. For $v_{h} \in Y^{h}$,

$$
\begin{aligned}
\left\|T_{1}^{\prime}\left(v_{h}\right)\right\|_{*} \equiv \sup _{\psi_{h} \in V_{0}^{h}, \psi_{h} \neq 0} \frac{\| T_{1}^{\prime}\left(v_{h}\right)\left(\psi_{h}\right)||}{\left|\psi_{h}\right|_{1}} \\
\quad \leq \sup _{\psi_{h} \in V_{0}^{h}, \psi_{h} \neq 0} \frac{\left|T_{1}^{\prime}\left(v_{h}\right)\left(\psi_{h}\right)\left(\psi_{h}\right)\right|}{\left|\psi_{h}\right|_{1}^{2}} \leq \frac{M-m}{M+m} .
\end{aligned}
$$

Proof. Let $\alpha=\sup _{\psi_{h} \in V_{0}^{h}, \psi_{h} \neq 0} \frac{\left|T_{1}^{\prime}\left(v_{h}\right)\left(\psi_{h}\right)\left(\psi_{h}\right)\right|}{\left|\psi_{h}\right|_{1}^{2}}$. We have

$$
\left|T_{1}^{\prime}\left(v_{h}\right)\left(\psi_{h}\right)\left(\psi_{h}\right)\right| \leq \alpha\left|\psi_{h}\right|_{1}^{2}, \psi_{h} \in V_{0}^{h}
$$

Since for $\mu_{h} \in V_{0}^{h},|| T_{1}^{\prime}\left(v_{h}\right)\left(\mu_{h}\right)||=\sup _{\eta_{h} \in V_{0}^{h}, \eta_{h} \neq 0}\left|T_{1}^{\prime}\left(v_{h}\right)\left(\mu_{h}\right)\left(\eta_{h}\right)\right| /\left|\eta_{h}\right|_{1}$, we obtain

$$
\left\|T_{1}^{\prime}\left(v_{h}\right)\right\|_{*}=\sup _{\mu_{h}, \eta_{h} \in V_{0}^{h}, \mu_{h}, \eta_{h} \neq 0} \frac{\left|T_{1}^{\prime}\left(v_{h}\right)\left(\mu_{h}\right)\left(\eta_{h}\right)\right|}{\left|\mu_{h}\right|{ }_{1}\left|\eta_{h}\right|_{1}} .
$$

But

$$
\begin{aligned}
T_{1}^{\prime}\left(v_{h}\right)\left(\mu_{h}\right)\left(\eta_{h}\right) & =\int_{\Omega} D \mu_{h} \cdot D \eta_{h} d x-\frac{1}{\nu} \int_{\Omega}\left[\left(\operatorname{cof} D^{2} v_{h}\right) D \mu_{h}\right] \cdot D \eta_{h} d x \\
& =\int_{\Omega}\left[\left(I-\frac{1}{\nu}\left(\operatorname{cof} D^{2} v_{h}\right)\right) D \mu_{h}\right] \cdot D \eta_{h} d x
\end{aligned}
$$

where $I$ denotes the $n \times n$ identity matrix. Hence

$$
\frac{T_{1}^{\prime}\left(v_{h}\right)\left(\mu_{h}\right)\left(\eta_{h}\right)}{\left|\mu_{h}\right|_{1}\left|\eta_{h}\right|_{1}}=\int_{\Omega}\left[\left(I-\frac{1}{\nu}\left(\operatorname{cof} D^{2} v_{h}\right)\right) D \frac{\mu_{h}}{\left|\mu_{h}\right|_{1}}\right] \cdot D \frac{\eta_{h}}{\left|\eta_{h}\right|_{1}} d x .
$$


Next, we note that for fixed $v_{h} \in Y^{h}$, we can define a bilinear form on $V_{0}^{h}$ by the formula

$$
(p, q)=\int_{\Omega}\left[\left(I-\frac{1}{\nu}\left(\operatorname{cof} D^{2} v_{h}\right)\right) D p\right] \cdot D q d x
$$

Then since

$$
(p, q)=\frac{1}{4}((p+q, p+q)-(p-q, p-q))
$$

we obtain

$$
\begin{gathered}
\left\|T_{1}^{\prime}\left(v_{h}\right)\right\|_{*}=\sup _{\mu_{h}, \eta_{h} \in V_{0}^{h}, \mu_{h}, \eta_{h} \neq 0} \frac{1}{4} \mid \int_{\Omega}\left[\left(I-\frac{1}{\nu} \operatorname{cof} D^{2} v_{h}\right)\right. \\
D\left(\frac{\mu_{h}}{\left|\mu_{h}\right|_{1}}+\frac{\eta_{h}}{\left|\eta_{h}\right|_{1}}\right] \cdot D\left(\frac{\mu_{h}}{\left|\mu_{h}\right|_{1}}+\frac{\eta_{h}}{\left|\eta_{h}\right|_{1}}\right) d x \\
\quad-\int_{\Omega}\left[\left(I-\frac{1}{\nu} \operatorname{cof} D^{2} v_{h}\right) D\left(\frac{\mu_{h}}{\left|\mu_{h}\right|_{1}}-\frac{\eta_{h}}{\left|\eta_{h}\right|_{1}}\right] \cdot D\left(\frac{\mu_{h}}{\left|\mu_{h}\right|_{1}}-\frac{\eta_{h}}{\left|\eta_{h}\right|_{1}}\right) d x \mid\right. \\
\leq \frac{\alpha}{4}\left(\left|\frac{\mu_{h}}{\left|\mu_{h}\right|_{1}}+\frac{\eta_{h}}{\left|\eta_{h}\right|_{1}}\right|_{1}^{2}+\left|\frac{\mu_{h}}{\left|\mu_{h}\right|_{1}}-\frac{\eta_{h}}{\left|\eta_{h}\right|_{1}}\right|_{1}^{2}\right)=\alpha .
\end{gathered}
$$

By Lemma 2.6 we have

$$
\left(1-\frac{M}{\nu}\right)|w|_{1}^{2} \leq \int_{\Omega}\left[\left(I-\frac{1}{\nu}\left(\operatorname{cof} D^{2} v_{h}\right)\right) D w\right] \cdot D w d x \leq\left(1-\frac{m}{\nu}\right)|w|_{1}^{2}, w \in H_{0}^{1}(\Omega) .
$$

Since $\nu=(M+m) / 2,1-M / \nu=-(M-m) /(M+m)$ and $1-m / \nu=(M-m) /(M+m)$, we conclude that $\alpha \leq(M-m) /(M+m)$.

We can now prove the following lemma

Lemma 4.2. The mapping $T_{1}$ is a strict contraction in $Y^{h}$ with contraction constant $(M-m) /(M+m)$ for $\nu=(M+m) / 2$.

Proof. Let $v_{h}$ and $w_{h} \in Y^{h}$. Then, using the Mean Value Theorem

$$
\begin{aligned}
\left\|T_{1}\left(w_{h}\right)-T_{1}\left(v_{h}\right)\right\| & =\left\|\int_{0}^{1} T_{1}^{\prime}\left(v_{h}+t\left(w_{h}-v_{h}\right)\right)\left(w_{h}-v_{h}\right) d t\right\| \\
& \leq \int_{0}^{1}\left\|T_{1}^{\prime}\left(v_{h}+t\left(w_{h}-v_{h}\right)\right)\left(w_{h}-v_{h}\right)\right\| d t .
\end{aligned}
$$

Since $w_{h}-v_{h} \in V_{0}^{h}$ and $v_{h}+t\left(w_{h}-v_{h}\right) \in Y^{h}, t \in[0,1]$, we obtain by Lemma 4.1.

$$
\left\|T_{1}\left(w_{h}\right)-T_{1}\left(v_{h}\right)\right\| \leq \int_{0}^{1} \frac{M-m}{M+m}\left|w_{h}-v_{h}\right|_{1} d t=\frac{M-m}{M+m}\left|w_{h}-v_{h}\right|_{1} .
$$

Remark 4.3. For the operator $T_{1}$ to be a strict contraction, it is enough to have $\nu$ sufficiently large, i.e. $\nu>M$. In our computations, the value of $\nu$ is chosen "adaptively", i.e. we start with the value $\nu=50$ and if necessary we reduce or increase it for better accuracy. The situation is similar to the setting of adaptive mesh refinements where it is not known in advance where to do a local refinement and decisions are made based on computed results. 
Remark 4.4. By the inverse inequality, we have

$$
C_{3} h^{2}\left|w_{h}\right|_{1}^{2} \leq \|\left. w_{h}\right|_{0} ^{2} \leq C_{1}^{2}\left|w_{h}\right|_{1}^{2}, w_{h} \in V_{0}^{h}
$$

We may assume that $C_{1} \leq 1$ by assuming that the domain is contained in a cube of side length at most 1. It follows that for $w_{h} \in V_{0}^{h}$

$$
\left(C_{3} h^{2}-\frac{M}{\nu}\right)\left|w_{h}\right|_{1}^{2} \leq \int_{\Omega} w_{h}^{2} d x-\int_{\Omega}\left[\frac{1}{\nu}\left(\operatorname{cof} D^{2} v_{h}\right) D w_{h}\right] \cdot D w_{h} d x \leq\left(1-\frac{m}{\nu}\right)\left|w_{h}\right|_{1}^{2} .
$$

As in the proofs of Lemmas 4.1 and 4.2, we conclude that for $\nu>M /\left(C_{3} h^{2}\right)$, the mapping $T_{2}: Y^{h} \rightarrow\left(V_{0}^{h}\right)^{\prime}$ defined by

$$
\left.<T_{2}\left(v_{h}\right), \psi_{h}\right\rangle=\int_{\Omega} v_{h} \psi_{h} d x+\frac{1}{\nu} \int_{\Omega}\left(\operatorname{det} D^{2} v_{h}-f\right) \psi_{h} d x
$$

for $v_{h} \in Y^{h}, \psi_{h} \in V_{0}^{h}$ is a strict contraction.

We can now claim our main result, which is the convergence to $u_{h}$ of the sequence defined by $u_{k+1} \in V^{h}, u_{k+1}=g_{h}$ on $\partial \Omega$ and

$$
\nu \int_{\Omega} D u_{k+1} \cdot D \psi_{h} d x=\nu \int_{\Omega} D u_{k} \cdot D \psi_{h} d x+\int_{\Omega}\left(\operatorname{det} D^{2} u_{k}-f\right) \psi_{h} d x,
$$

for $\psi_{h} \in V_{0}^{h}$.

Theorem 4.5. Let $\Omega$ be convex with a Lipschitz continuous boundary and assume that the spaces $V^{h}=S_{d}^{r}(\mathcal{T})$ have the optimal approximation property 2.2 and satisfy the inverse estimates (2.4). The sequence defined by (4.4) converges to the unique strictly convex solution $u_{h}$ of (2.8) for any initial guess $u_{0}$ in $Y^{h}$ and a suitable $\nu>0$ with a linear convergence rate.

Proof. The proof parallels Theorem 5.4 in [18]. Let us assume first that $u_{k} \in Y^{h}$. We have using (2.8), or equivalently $\operatorname{det} D^{2} u_{h}=f$ in $\left(V_{0}^{h}\right)^{\prime}$,

$$
\begin{aligned}
\int_{\Omega} D\left(u_{k+1}-u_{h}\right) \cdot D \psi_{h} d x & =\int_{\Omega} D\left(u_{k}-u_{h}\right) \cdot D \psi_{h} d x+\frac{1}{\nu} \int_{\Omega} \operatorname{det} D^{2} u_{k} \psi_{h} d x \\
& -\frac{1}{\nu} \int_{\Omega} \operatorname{det} D^{2} u_{h} \psi_{h} d x \\
& =\left\langle T_{1}\left(u_{k}\right)-T_{1}\left(u_{h}\right), \psi_{h}\right\rangle .
\end{aligned}
$$

Taking $\psi_{h}=u_{k+1}-u_{h}$, we obtain

$$
\left|u_{k+1}-u_{h}\right|_{1}^{2} \leq|| T_{1}\left(u_{k}\right)-T_{1}\left(u_{h}\right)||\left|u_{k+1}-u_{h}\right|_{1} \leq \frac{M-m}{M+m}\left|u_{k}-u_{h}\right|_{1}\left|u_{k+1}-u_{h}\right|_{1},
$$

where for simplicity, we assume that the finite dimensional $V_{0}^{h}$ is equipped with the |.| 1 norm of $H_{0}^{1}(\Omega)$. We conclude that

$$
\left|u_{k+1}-u_{h}\right|_{1} \leq \frac{M-m}{M+m}\left|u_{k}-u_{h}\right|_{1}
$$

This also shows that if $u_{k} \in Y^{h}$, then $u_{k+1} \in Y^{h}$ and concludes the proof. 
Remark 4.6. It follows from the above result and Remark 4.4 that for a suitable initial guess and a suitable $\nu>0$, the sequence defined by

$$
\nu \int_{\Omega} u_{k+1} \psi_{h} d x=\nu \int_{\Omega} u_{k} \psi_{h} d x+\int_{\Omega}\left(\operatorname{det} D^{2} u_{k}-f\right) \psi_{h} d x, \psi_{h} \in V_{0}^{h},
$$

with $u_{k+1} \in V^{h}, u_{k+1}=g_{h}$ on $\partial \Omega$ also converges to the unique strictly convex solution of 2.8. Obviously the convergence properties of (4.4) and (4.5) depend on the contraction constants of $T_{1}$ and $T_{2}$ respectively. Thus (4.4) is more robust than (4.5) in the sense that the choice of $\nu$ for (4.4) is less dependent on the discretization parameter $h$. As suggested in [23] in the context of monotone schemes, the use of the Laplacian preconditioner results in a more efficient algorithm.

\section{Numerical Results}

The numerical results are obtained with the spline element method which we first review. We conclude the section with some heuristics about why our methods appear to enforce convexity.

5.1. Spline element discretization. We refer to [1, 4, 5, 7, 25, 2, for a description of the spline element method. We describe the method for linear problems and recall that the problems (1.3) are linear problems. Let $u \in V=H_{0}^{m}(\Omega), m \geq 1$ solve a variational problem $a(u, v)=f(v)$ with the conditions of the Lax-Milgram lemma satisfied. Take $V_{h}$ as the spline space $S_{d}^{r}(\mathcal{T})$ of smoothness $r$ and degree $d,(2.1)$. For $r=0$ and $d=1$ we have the space of piecewise linear continuous functions.

First, start with a representation of a piecewise discontinuous polynomial as a vector in $\mathbb{R}^{N}$, for some integer $N>0$. Then express boundary conditions and constraints including global continuity or smoothness conditions as linear relations. In our work, we use the Bernstein basis representation, [1, 2] which is very convenient to express smoothness conditions and very popular in computer aided geometric design. Hence the term "spline" in the name of the method. We can therefore identify the space $V_{h}$ with $\left\{c \in \mathbb{R}^{N}, R c=G\right\}$ for some integer $N$, matrix $R$ and vector $G$. The discrete problem consists in finding $c \in V_{h}, c^{T} K d=F^{T} d$ for all $d \in V_{h}$ for a suitable stiffness matrix $K$ and a load vector $F$. Introducing a Lagrange multiplier $\lambda$, the functional

$$
K(c) d-L^{T} d+\lambda^{T} R d,
$$

vanishes identically on $V_{h}$. The stronger condition

$$
K(c)+\lambda^{T} R=L^{T},
$$

along with the side condition $R c=G$ are the discrete equations to be solved. We are lead to saddle point problems

$$
\left(\begin{array}{cc}
K & R^{T} \\
R & 0
\end{array}\right)\left(\begin{array}{l}
\mathbf{c} \\
\lambda
\end{array}\right)=\left[\begin{array}{l}
F \\
G
\end{array}\right] .
$$

The ellipticity condition assures uniqueness of the component $c$ and the saddle point problems are solved by a version of the augmented Lagrangian algorithm

$$
\left(K+\frac{1}{\mu} R^{T} R\right) c^{(l+1)}=K^{T} c^{(l)}+\frac{1}{\mu} R^{T} G, \quad l=1,2, \ldots
$$




\begin{tabular}{|c|c|c|c|c|c|c|c|}
\hline$h$ & $n_{i t}$ & $L^{2}$ norm & rate & $H^{1}$ norm & rate & $H^{2}$ norm & rate \\
\hline $1 / 2^{1}$ & 236 & $4.156910^{-6}$ & & $6.514210^{-5}$ & & $1.936410^{-3}$ & \\
\hline $1 / 2^{2}$ & 233 & $1.150410^{-7}$ & 5.17 & $2.391510^{-6}$ & 4.77 & $1.344410^{-4}$ & 3.85 \\
\hline $1 / 2^{3}$ & 233 & $3.240610^{-9}$ & 5.15 & $8.412010^{-8}$ & 4.83 & $8.936610^{-6}$ & 3.92 \\
\hline $1 / 2^{4}$ & 233 & $4.585710^{-10}$ & 2.82 & $4.724610^{-9}$ & 4.15 & $6.070610^{-7}$ & 3.88 \\
\hline
\end{tabular}

TABLE 1. Time marching method for Test $1, S_{5}^{1}, \nu=50$

\begin{tabular}{|c|c|c|c|c|}
\hline d & $n_{i t}$ & $L^{2}$ norm & $H^{1}$ norm & $H^{2}$ norm \\
\hline 3 & 1 & $1.233810^{-2}$ & $7.698410^{-2}$ & $4.441110^{-1}$ \\
\hline 4 & 270 & $1.628910^{-3}$ & $1.471910^{-2}$ & $1.398310^{-1}$ \\
\hline 5 & 135 & $1.533310^{-3}$ & $8.731210^{-3}$ & $6.041210^{-2}$ \\
\hline 6 & 424 & $1.249110^{-4}$ & $9.745810^{-4}$ & $1.047310^{-2}$ \\
\hline Rate & & $0.180 .25^{d-1}$ & $4.570 .25^{d}$ & $60.850 .3^{d+1}$ \\
\hline
\end{tabular}

TABLE 2. Time marching method for Test $2(3 \mathrm{D})$ on $\mathcal{I}_{1}, \nu=50$

The convergence properties of the iterative method were given in [6]. Extensive implementation details can be found in [1, 5].

5.2. Numerical results. For $n=2$, the computational domain is the unit square $[0,1]^{2}$ which is first divided into squares of side length $h$. Then each square is divided into two triangles by the diagonal with negative slope. For $n=3$, the initial tetrahedral partition $\mathcal{I}_{1}$ consists in six tetrahedra. Each tetrahedron is then uniformly refined into 8 subtetrahedra forming $\mathcal{I}_{2}$. In the tables, $n_{i t}$ denotes the number of iterations. We refer to [1, 5] for implementation details of the method. All numerical experiments are with the versions of the iterative methods with Laplacian preconditioner.

In general we did not try to choose the value of $\nu$ that would give the smallest number of iterations except in Tables 4 and 5 where we compare the performance of the two methods.

We use some standard test cases for numerical evidence for convergence to non smooth solutions of the elliptic Monge-Ampere equation.

Test 1: $u(x, y)=e^{\left(x^{2}+y^{2}\right) / 2}$ so that $f(x, y)=\left(1+x^{2}+y^{2}\right) e^{\left(x^{2}+y^{2}\right)}$ and $g(x, y)=$ $e^{\left(x^{2}+y^{2}\right) / 2}$ on $\partial \Omega$.

Test 2: $u(x, y, z)=e^{\left(x^{2}+y^{2}+z^{2}\right) / 3}$ so that $f(x, y, z)=8 / 81\left(3+2\left(x^{2}+y^{2}+z^{2}\right) e^{\left(x^{2}+y^{2}+z^{2}\right)}\right.$ and $g(x, y, z)=e^{\left(x^{2}+y^{2}+z^{2}\right) / 3}$ on $\partial \Omega$.

Barring roundoff errors, the methods introduced in this paper capture smooth solutions. For the two dimensional test function, Test 1, we give numerical results for successive refinements and for the three dimensional test function, we give numerical results for increasing values of the degree $d$ on two successive refinements.

In the context of approximations by finite dimensional spaces, many finite element methods proposed, [15, 21], fail to fully capture the convexity of the solution on the test case 


\begin{tabular}{|c|c|c|c|c|}
\hline $\mathrm{d}$ & $n_{i t}$ & $L^{2}$ norm & $H^{1}$ norm & $H^{2}$ norm \\
\hline 3 & 1 & $3.173910^{-3}$ & $2.300510^{-2}$ & $2.4496 \quad 10^{-1}$ \\
\hline 4 & 651 & $3.238510^{-4}$ & $3.559910^{-3}$ & $5.2262 \quad 10^{-2}$ \\
\hline 5 & 744 & $2.273010^{-5}$ & $3.897710^{-4}$ & $8.897810^{-3}$ \\
\hline 6 & 652 & $1.195610^{-6}$ & $2.205610^{-5}$ & $6.043710^{-4}$ \\
\hline Rate & & $0.720 .072^{d-1}$ & $29.440^{d}$ & $861.430 .14^{d+1}$ \\
\hline
\end{tabular}

TABLE 3. Time marching method for Test $2(3 \mathrm{D})$ on $\mathcal{I}_{2}, \nu=50$

\begin{tabular}{|c|c|c|c|c|c|}
\hline$h$ & $\nu$ & $n_{i t}$ & time & $L^{2}$ norm & rate \\
\hline $1 / 2^{1}$ & 0 & 6 & $3.032810^{+0}$ & $2.195410^{-2}$ & \\
\hline $1 / 2^{2}$ & 0 & 5 & $8.136510^{+0}$ & $3.609710^{-3}$ & 2.60 \\
\hline $1 / 2^{3}$ & 0 & 6 & $3.823010^{+1}$ & $1.068510^{-3}$ & 1.76 \\
\hline $1 / 2^{4}$ & 3 & 56 & $1.597910^{+3}$ & $3.766610^{-4}$ & 1.50 \\
\hline
\end{tabular}

TABle 4. Pseudo-transient method Test $4 r=1, d=3$

\begin{tabular}{|c|c|c|c|c|c|}
\hline$h$ & $\nu$ & $n_{i t}$ & time & $L^{2}$ norm & rate \\
\hline $1 / 2^{1}$ & 2 & 35 & $6.219110^{+0}$ & $2.072110^{-2}$ & \\
\hline $1 / 2^{2}$ & 2 & 89 & $6.055310^{+1}$ & $1.857910^{-3}$ & 3.48 \\
\hline $1 / 2^{3}$ & 4.5 & 64 & $1.684910^{+2}$ & $5.043810^{-4}$ & 1.88 \\
\hline $1 / 2^{4}$ & 11.5 & 151 & $1.703810^{+3}$ & $2.113210^{-4}$ & 1.25 \\
\hline
\end{tabular}

TABLE 5. Time marching method Test $4 r=1, d=3$

Test 3: $g(x, y)=0$ and $f(x, y)=1$.

In Figure 1 we give a plot of the graph of the solution as well as a section of the graph along the line $y=x$.

For the same test case, there is a concave solution. The concavity property of the concave solution obtained with the time marching method are better than the one obtained by the vanishing moment methodology, [21]. This is illustrated in Figure 4 .

We now discuss how the two methods compare. First, we are solving the same discrete equations (2.8) by different iterative methods. Second, we noticed that the smaller $\nu$, the smaller the number of iterations. Thus for a smooth solution, the correct value of $\nu$ to take in the pseudo transient method is $\nu=0$ which is exactly Newton's method. In fact, Newton's method has been shown to have a quadratic convergence rate while the pseudo transient methods and time marching methods are shown in Theorems 3.1 and 4.5 to have a linear convergence rate. Moreover the numerical errors of Tables 1, 2 and 3 are essentially the ones obtained with Newton's method as expected. We compare the performance of the methods on a non-smooth solution with known solution.

Test 4: $u(x, y)=-\sqrt{2-x^{2}-y^{2}}$ with corresponding $f$ and $g$. 

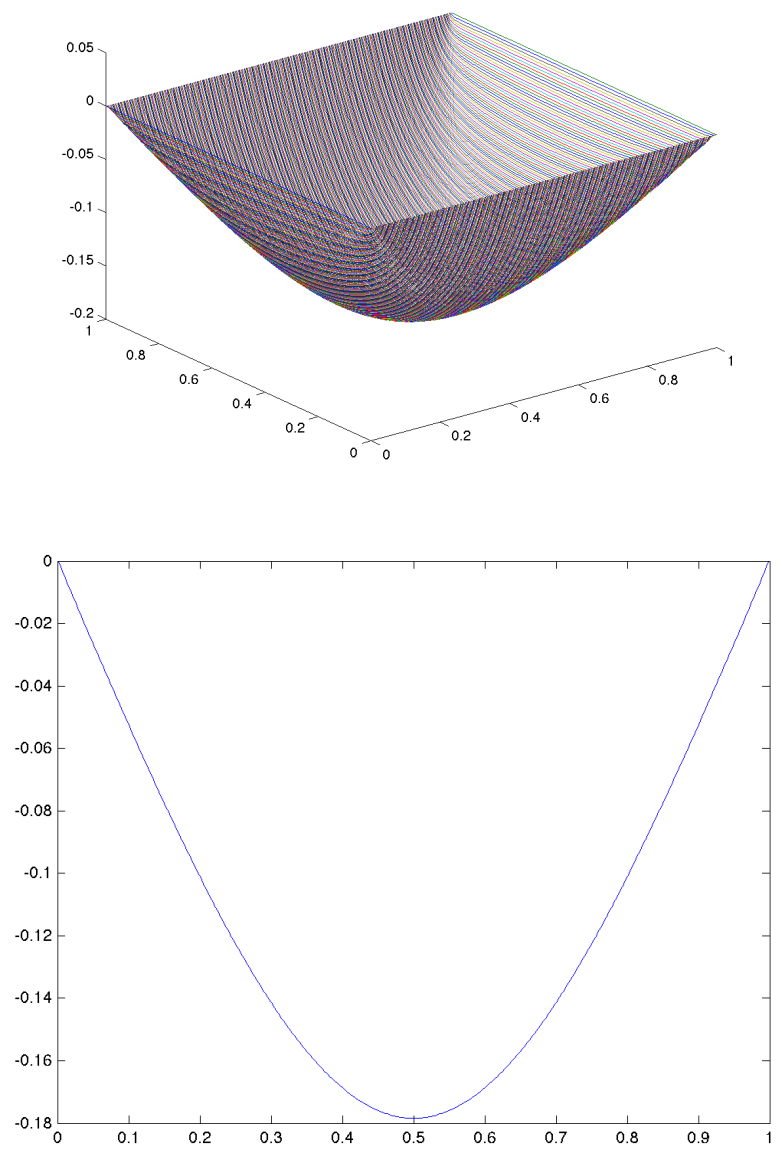

Figure 1. Pseudo transient Test 3, convex solution: $h=1 / 2^{4}, d=$ $5, \nu=7.5$.

The time listed is in seconds and obtained on an imac running Mac OS 10.6.8 with a $2.4 \mathrm{Ghz}$ intel core 2 duo and 4 GB of SDRAM memory. While for small values of $h$ the time marching method appears to take significantly more time, it is also significantly more accurate. For $h=1 / 2^{4}$ the time took by the two methods is almost the same with the time marching method giving a more accurate solution.

Next we consider a non square domain.

Test 5: we consider the unit circle discretized with a Delanauy triangulation with 824 triangles and $u(x, y)=x^{2}+y^{2}-1$ which vanishes on the boundary, Figure 3 .

We conclude this section with a test problem for a degenerate Monge-Ampère equation Test $6: g(x, y)=|x-1 / 2|$ and $f(x, y)=0$.

The graph of the function, Figure 4 is singular along the line $x=1 / 2$. The approximations been $C^{1}$ do appear to capture the singularity but not the convexity of the solution. Somewhat better results are obtained with another iterative method discussed in an unpublished report available at http://arxiv.org/abs/1012.1775. When 

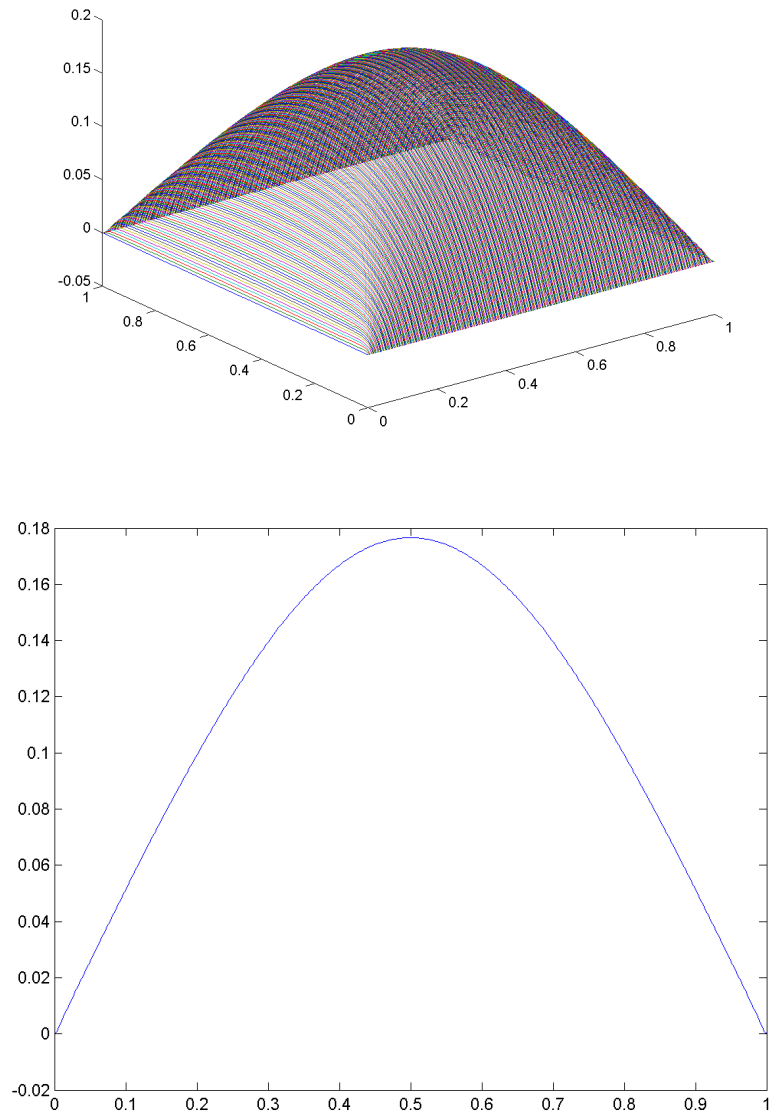

Figure 2. Time marching, Test 3 , concave solution: $h=1 / 2^{4}, d=$ $5, \nu=50$.

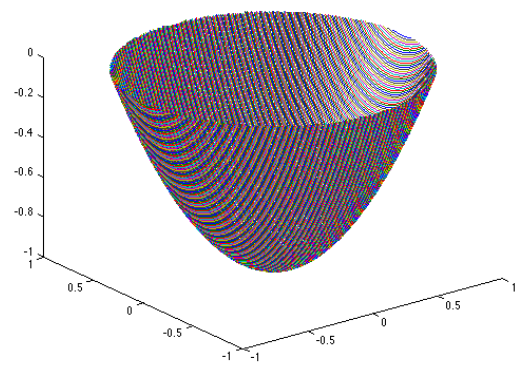

Figure 3. $u(x, y)=x^{2}+y^{2}-1$ on a non square domain with pseudo transient $\nu=0, r=1, d=3$ 

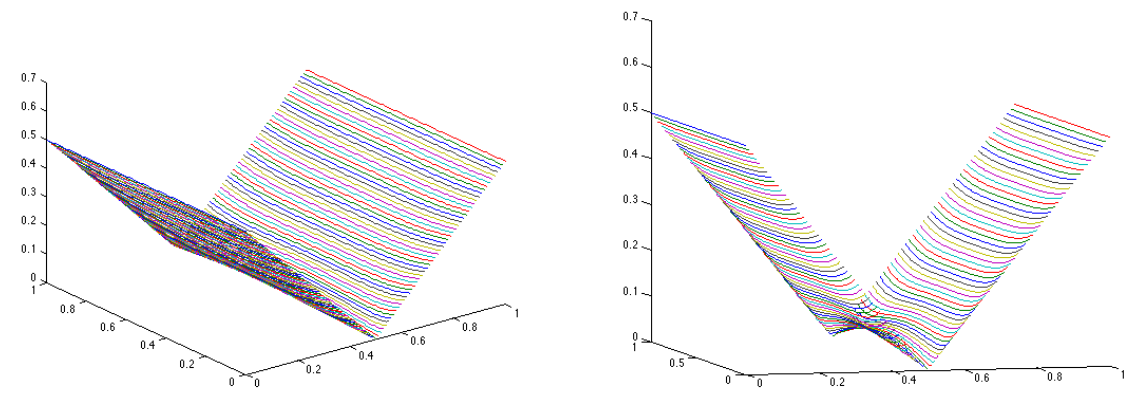

FiguRE 4. $f(x, y)=0$ and $g(x, y)=|x-1 / 2|$ with time marching $\nu=50, r=1, d=5, h=1 / 2^{4}$

the time marching method is discretized by the standard finite difference method the singularity is captured correctly. We wish to discuss these results in separate works.

5.3. Heuristics on convexity preservation. When (1.1) has a smooth strictly convex solution, Theorem 2.2 establishes that the approximate solution is automatically convex. The numerical experiments indicate that in the non smooth case, discrete solutions are also convex. The result can be easily explained at the continuous level (for a smooth solution).

Assume that $f>c_{0}>0$ and that the sequence $u_{k}$ defined by

$$
\begin{aligned}
\nu \Delta u_{k+1}+\left(\operatorname{cof} D^{2} u_{k}\right): D^{2} u_{k+1}=\nu \Delta u_{k} & +\left(\operatorname{cof} D^{2} u_{k}\right): D^{2} u_{k} \\
& -\operatorname{det} D^{2} u_{k}+f .
\end{aligned}
$$

has been shown to converge to $u$ in the Hölder space $C^{2, \beta}(\Omega)$ for some $\beta$ in $(0,1)$. From the arithmetic-geometric inequality, we have

$$
\frac{\left(\Delta u_{k}\right)^{n}}{n^{n}} \geq \operatorname{det} D^{2} u_{k}
$$

By the continuity of the eigenvalues, (2.13), $\Delta v$ is bounded in a neighborhood of $u$ in which all $u_{k}$ belong for $k$ large enough. Choose $\nu$ such that $\nu \geq(n-1)\left(\Delta u_{k}\right)^{n-1} / n^{n}$ for all $k$ and note that the right hand of $(5.2)$ is equal to $\nu \Delta u_{k}+(n-1) \operatorname{det} D^{2} u_{k}+f$. By the assumption on $\nu$, we get $\nu \Delta u_{k+1}+\left(\operatorname{cof} D^{2} u_{k}\right): D^{2} u_{k+1} \geq 0$. In the limit, we obtain $\nu \Delta u+\left(\operatorname{cof} D^{2} u\right): D^{2} u \geq 0$. Since det $D^{2} u \geq 0$ by assumption, we get $\Delta u \geq 0$.

As for the time marching method

$$
-\nu \Delta u_{k+1}=-\nu \Delta u_{k}+\operatorname{det} D^{2} u_{k}-f, u_{k+1}=g \text { on } \partial \Omega,
$$

assume now again that $f>c_{0}>0$ and that the sequence $u_{k}$ has been shown to converge to $u$ in $C^{2, \beta}(\Omega)$ for some $\beta$ in $(0,1)$. Choose $\nu$ such that $\nu \geq\left(\Delta u_{k}\right)^{n-1} / n^{n}$. It follows from the arithmetic-geometric inequality that

$$
\nu \Delta u_{k} \geq \frac{\left(\Delta u_{k}\right)^{n}}{n^{n}} \geq \operatorname{det} D^{2} u_{k}
$$

and so $-\nu \Delta u_{k}+\operatorname{det} D^{2} u_{k} \leq 0$ and it follows that the time marching method also preserves the positivity of the Laplacian. 
In two dimensions $\Delta u \geq 0$ and $\operatorname{det} D^{2} u=f \geq 0$ imply that $D^{2} u$ is positive.

\section{ACKNOWLEDGEMENTS}

The author would like to thank the referees for a careful reading of the paper and suggestions which led to a better presentation of the paper.

The author acknowledges discussions with F. Celiker, B. Cockburn, W. Gangbo, R. Glowinski, M.J. Lai, R. Nochetto, A. Oberman and A. Regev. The author was supported in part by NSF grants DMS-0811052, DMS-1319640 and the Sloan Foundation. This research was supported in part by the Institute for Mathematics and its Applications and the Mathematical Sciences Research Institute with funds provided by the National Science Foundation.

\section{REFERENCES}

[1] Awanou, G.: Energy methods in 3D spline approximations of the Navier-Stokes equations. Ph.D. Dissertation, University of Georgia. Athens, Ga (2003)

[2] Awanou, G.: Robustness of a spline element method with constraints. J. Sci. Comput. 36(3), 421-432 (2008)

[3] Awanou, G.: Standard finite elements for the numerical resolution of the elliptic Monge-Ampère equation: classical solutions (2014). To appear in IMA J. of Num. Analysis

[4] Awanou, G., Lai, M.J.: Trivariate spline approximations of 3D Navier-Stokes equations. Math. Comp. 74(250), 585-601 (electronic) (2005)

[5] Awanou, G., Lai, M.J., Wenston, P.: The multivariate spline method for scattered data fitting and numerical solution of partial differential equations. In: Wavelets and splines: Athens 2005, Mod. Methods Math., pp. 24-74. Nashboro Press, Brentwood, TN (2006)

[6] Awanou, G.M., Lai, M.J.: On convergence rate of the augmented Lagrangian algorithm for nonsymmetric saddle point problems. Appl. Numer. Math. 54(2), 122-134 (2005)

[7] Baramidze, V., Lai, M.J.: Spherical spline solution to a PDE on the sphere. In: Wavelets and splines: Athens 2005, Mod. Methods Math., pp. 75-92. Nashboro Press, Brentwood, TN (2006)

[8] Benamou, J.D., Brenier, Y.: A computational fluid mechanics solution to the MongeKantorovich mass transfer problem. Numer. Math. 84(3), 375-393 (2000)

[9] Böhmer, K.: On finite element methods for fully nonlinear elliptic equations of second order. SIAM J. Numer. Anal. 46(3), 1212-1249 (2008)

[10] Bohmer, K.: Numerical methods for nonlinear elliptic differential equations: a synopsis. Oxford University Press, USA (2010)

[11] Braess, D.: Finite elements, third edn. Cambridge University Press, Cambridge (2007). Theory, fast solvers, and applications in elasticity theory, Translated from the German by Larry L. Schumaker

[12] Brenner, S.C., Gudi, T., Neilan, M., Sung, L.Y.: $C^{0}$ penalty methods for the fully nonlinear Monge-Ampère equation. Math. Comp. 80(276), 1979-1995 (2011)

[13] Brenner, S.C., Neilan, M.: Finite element approximations of the three dimensional MongeAmpère equation. ESAIM Math. Model. Numer. Anal. 46(5), 979-1001 (2012)

[14] Brenner, S.C., Scott, L.R.: The mathematical theory of finite element methods, Texts in Applied Mathematics, vol. 15, second edn. Springer-Verlag, New York (2002)

[15] Dean, E.J., Glowinski, R.: Numerical methods for fully nonlinear elliptic equations of the Monge-Ampère type. Comput. Methods Appl. Mech. Engrg. 195(13-16), 1344-1386 (2006)

[16] Dyer, B.W., Hong, D.: Algorithm for optimal triangulations in scattered data representation and implementation. J. Comput. Anal. Appl. 5(1), 25-43 (2003). Approximation theory and wavelets (Austin, TX, 1999)

[17] Evans, L.C.: Partial differential equations, Graduate Studies in Mathematics, vol. 19. American Mathematical Society, Providence, RI (1998) 
[18] Faragó, I., Karátson, J.: Numerical solution of nonlinear elliptic problems via preconditioning operators: theory and applications, Advances in Computation: Theory and Practice, vol. 11. Nova Science Publishers Inc., Hauppauge, NY (2002)

[19] Feng, X., Glowinski, R., Neilan, M.: Recent Developments in Numerical Methods for Fully Nonlinear Second Order Partial Differential Equations. SIAM Rev. 55(2), 205-267 (2013)

[20] Feng, X., Neilan, M.: Convergence of a fourth order singular perturbation of the $n$-dimensional radially symmetric Monge-Ampere equation. To appear in Applicable Analysis

[21] Feng, X., Neilan, M.: Vanishing moment method and moment solutions for second order fully nonlinear partial differential equations. J. Sci. Comput. 38(1), 74-98 (2009)

[22] Feng, X., Neilan, M.: Analysis of Galerkin methods for the fully nonlinear Monge-Ampère equation. J. Sci. Comput. 47(3), 303-327 (2011)

[23] Glowinski, R.: Numerical methods for fully nonlinear elliptic equations. In: ICIAM 07-6th International Congress on Industrial and Applied Mathematics, pp. 155-192. Eur. Math. Soc., Zürich (2009)

[24] Hoffman, A.J., Wielandt, H.W.: The variation of the spectrum of a normal matrix. Duke Math. J. 20, 37-39 (1953)

[25] Hu, X.L., Han, D.F., Lai, M.J.: Bivariate splines of various degrees for numerical solution of partial differential equations. SIAM J. Sci. Comput. 29(3), 1338-1354 (electronic) (2007)

[26] Kelley, C.T., Keyes, D.E.: Convergence analysis of pseudo-transient continuation. SIAM J. Numer. Anal. 35(2), 508-523 (1998)

[27] Lai, M.J.: Convex preserving scattered data interpolation using bivariate $C^{1}$ cubic splines. J. Comput. Appl. Math. 119(1-2), 249-258 (2000)

[28] Lai, M.J., Schumaker, L.L.: Spline functions on triangulations, Encyclopedia of Mathematics and its Applications, vol. 110. Cambridge University Press, Cambridge (2007)

[29] Loeper, G., Rapetti, F.: Numerical solution of the Monge-Ampère equation by a Newton's algorithm. C. R. Math. Acad. Sci. Paris 340(4), 319-324 (2005)

[30] Oberman, A.M.: Wide stencil finite difference schemes for the elliptic Monge-Ampère equation and functions of the eigenvalues of the Hessian. Discrete Contin. Dyn. Syst. Ser. B 10(1), 221-238 (2008)

[31] Oliker, V.I., Prussner, L.D.: On the numerical solution of the equation $\left(\partial^{2} z / \partial x^{2}\right)\left(\partial^{2} z / \partial y^{2}\right)-$ $\left(\left(\partial^{2} z / \partial x \partial y\right)\right)^{2}=f$ and its discretizations. I. Numer. Math. 54(3), 271-293 (1988)

Department of Mathematics, Statistics, and Computer Science, M/C 249. University of Illinois at Chicago, Chicago, IL 60607-7045, USA

E-mail address: awanou@uic.edu

$U R L:$ http: //www.math.uic.edu/ awanou 